\title{
VANISHING CYCLES, THE GENERALIZED HODGE CONJECTURE AND GRÖBNER BASES
}

\author{
ICHIRO SHIMADA \\ Division of Mathematics, Graduate School of Science, Hokkaido University \\ Sapporo 060-0810, Japan \\ E-mail: shimada@math.sci.hokudai.ac.jp
}

\begin{abstract}
Let $X$ be a general complete intersection of a given multi-degree in a complex projective space. Suppose that the anti-canonical line bundle of $X$ is ample. Using the cylinder homomorphism associated with the family of complete intersections of a smaller multi-degree contained in $X$, we prove that the vanishing cycles in the middle homology group of $X$ are represented by topological cycles whose support is contained in a proper Zariski closed subset $T$ of $X$ with certain codimension. In some cases, by means of Gröbner bases, we can find such a Zariski closed subset $T$ with codimension equal to the upper bound obtained from the Hodge structure of the middle cohomology group of $X$. Hence a consequence of the generalized Hodge conjecture is verified in these cases.
\end{abstract}

1. Introduction. There are only few non-trivial examples that can be used as supporting evidence for the generalized Hodge conjecture formulated by Grothendieck [8]. In this paper, we deal with complete intersections of small multi-degrees in a complex projective space, and prove, in some cases, a consequence of the generalized Hodge conjecture for these complete intersections by means of cylinder homomorphisms.

We work over the complex number field $\mathbb{C}$. Let $X$ be a general complete intersection of multi-degree $\mathbf{a}=\left(a_{1}, \ldots, a_{r}\right)$ in $\mathbb{P}^{n}$ with $\min (\mathbf{a}) \geq 2$. Suppose that $X$ is Fano, that is, the total degree $\sum_{i=1}^{r} a_{i}$ of $X$ is less than or equal to $n$. We put

$$
m:=\operatorname{dim} X=n-r \quad \text { and } \quad k:=\left[\frac{1}{\max (\mathbf{a})}\left(n-\sum_{i=1}^{r} a_{i}\right)\right]+1,
$$

where [ ] denotes the integer part. It is known that the Hodge structure of the middle

2000 Mathematics Subject Classification: Primary 14C30, 14M10; Secondary 14C05, 14J45, $14 \mathrm{~J} 05$.

The paper is in final form and no version of it will be published elsewhere. 
cohomology group $H^{m}(X, \mathbb{Q})$ of $X$ satisfies the following ([6, Corollaire 2.8]):

$$
H^{\nu, m-\nu}(X)=0 \Longleftrightarrow 0 \leq \nu<k \text { or } 0 \leq m-\nu<k .
$$

If the generalized Hodge conjecture is true, then there should exist a Zariski closed subset $T$ of $X$ with codimension $k$ such that the inclusion $T \hookrightarrow X$ induces a surjective homomorphism $H_{m}(T, \mathbb{Q}) \rightarrow H_{m}(X, \mathbb{Q})$.

We will try to verify this consequence of the generalized Hodge conjecture by means of cylinder homomorphisms. Let $\mathbf{b}=\left(b_{1}, \ldots, b_{s}\right)$ be another sequence of integers satisfying $\min (\mathbf{b}) \geq 1$ and $r<s<n$. We denote by $F_{\mathbf{b}}(X)$ the scheme parametrizing all complete intersections of multi-degree $\mathbf{b}$ in $\mathbb{P}^{n}$ that are contained in $X$, and by $Z_{\mathbf{b}}(X) \subset X \times F_{\mathbf{b}}(X)$ the universal family with

$$
\begin{aligned}
& Z_{\mathbf{b}}(X) \stackrel{\alpha_{X}}{\longrightarrow} X \\
& \pi_{X} \downarrow \\
& F_{\mathbf{b}}(X)
\end{aligned}
$$

being the diagram of the projections. We put

$$
l:=n-s .
$$

Suppose that $F_{\mathbf{b}}(X)$ is non-empty, and that $m>2 l$ holds. Since $\pi_{X}$ is proper and flat of relative dimension $l$, we have a homomorphism

$$
H_{m-2 l}\left(F_{\mathbf{b}}(X), \mathbb{Z}\right) \rightarrow H_{m}\left(Z_{\mathbf{b}}(X), \mathbb{Z}\right)
$$

that maps a homology class $[\tau] \in H_{m-2 l}\left(F_{\mathbf{b}}(X), \mathbb{Z}\right)$ represented by a topological $(m-2 l)$ cycle $\tau$ in $F_{\mathbf{b}}(X)$ to the homology class $\left[\pi_{X}^{-1}(\tau)\right] \in H_{m}\left(Z_{\mathbf{b}}(X), \mathbb{Z}\right)$ represented by the topological $m$-cycle $\pi_{X}^{-1}(\tau)$ in $Z_{\mathbf{b}}(X)$. We define a homomorphism

$$
\psi_{\mathbf{b}}(X): H_{m-2 l}\left(F_{\mathbf{b}}(X), \mathbb{Z}\right) \rightarrow H_{m}(X, \mathbb{Z})
$$

by

$$
\psi_{\mathbf{b}}(X)([\tau]):=\alpha_{X *}\left(\left[\pi_{X}^{-1}(\tau)\right]\right),
$$

and call $\psi_{\mathbf{b}}(X)$ the cylinder homomorphism associated with the family of algebraic cycles $\pi_{X}: Z_{\mathbf{b}}(X) \rightarrow F_{\mathbf{b}}(X)$.

It was remarked in [18] that there exists a Zariski closed subset $T$ of $X$ with codimension greater than or equal to $l$ such that the image of the homomorphism $H_{m}(T, \mathbb{Q}) \rightarrow$ $H_{m}(X, \mathbb{Q})$ induced from the inclusion $T \hookrightarrow X$ contains $\operatorname{Im} \psi_{\mathbf{b}}(X) \otimes \mathbb{Q}$. (See also Corollary 5.4 of this paper.) Therefore, in view of the generalized Hodge conjecture, it is an interesting problem to find a sequence $\mathbf{b}$ with $l$ as large as possible (hopefully $l=k$ ) such that the cylinder homomorphism $\psi_{\mathbf{b}}(X)$ has a "big" image.

Our Main Theorem, which will be stated in Section 2, gives us a sufficient condition on $(n, \mathbf{a}, \mathbf{b})$ for the image of $\psi_{\mathbf{b}}(X)$ to contain the module of vanishing cycles

$$
V_{m}(X, \mathbb{Z}):=\operatorname{Ker}\left(H_{m}(X, \mathbb{Z}) \rightarrow H_{m}\left(\mathbb{P}^{n}, \mathbb{Z}\right)\right)
$$

This sufficient condition can be checked by means of Gröbner bases. Combining Main Theorem with a theorem of Debarre and Manivel [5, Théorème 2.1] about the variety of linear subspaces contained in a general complete intersection, we also give a simple 
numerical condition on $(n, \mathbf{a}, \mathbf{b})$ that is sufficient for $\operatorname{Im} \psi_{\mathbf{b}}(X) \supseteq V_{m}(X, \mathbb{Z})$ to hold (Theorem 7.2). In many cases, our method yields $\mathbf{b}$ with $l$ larger than any previously known results, and sometimes we can verify the consequence of the generalized Hodge conjecture. See Section 8 for the examples.

After the work of Clemens and Griffiths [2] on the family of lines in a cubic threefold, many authors have studied the cylinder homomorphisms of type $\psi_{\mathbf{b}}(X)$, and proved that the image contains the module of vanishing cycles ([1], [3], [4], [10], [11], [12], [13], [14], [15], [16], [19], [21]). Our method provides us with a unified proof and a generalization of these results.

This paper is organized as follows. In Section 2, we state Main Theorem. In Section 3, we study a connection between vanishing cycles and cylinder homomorphisms in general setting. Theorem 3.1 in this section is essentially same as the result of [17]. However we present a complete and improved proof for readers' convenience. In Section 4, we construct the universal family of the families $Z_{\mathbf{b}}(X) \rightarrow F_{\mathbf{b}}(X)$ over the scheme parametrizing all complete intersections of multi-degree $\mathbf{a}$ in $\mathbb{P}^{n}$, which is a Zariski open subset of a Hilbert scheme, and study its properties. Combining the results of Sections 3 and 4, we prove Main Theorem in Section 5. In Section 6, we explain a method for checking the conditions on $(n, \mathbf{a}, \mathbf{b})$ required by Main Theorem by means of Gröbner bases. In Section 7, an application of the theorem of Debarre and Manivel is presented. Examples are investigated in relation to the generalized Hodge conjecture in Section 8.

\section{Conventions}

(1) We work over $\mathbb{C}$. A point of a scheme means a $\mathbb{C}$-valued point unless otherwise stated.

(2) For an analytic space $X$ or a scheme $X$ over $\mathbb{C}$, let $T_{p} X$ denote the Zariski tangent space to $X$ at a point $p$ of $X$.

(3) The multi-degree of a complete intersection is always denoted in the non-decreasing order.

2. Statement of Main Theorem. We fix an integer $n \geq 4$. Let $\mathbf{a}=\left(a_{1}, \ldots, a_{r}\right)$ and $\mathbf{b}=\left(b_{1}, \ldots, b_{s}\right)$ be sequences of integers satisfying

$$
2 \leq a_{1} \leq \ldots \leq a_{r}, \quad 1 \leq b_{1} \leq \ldots \leq b_{s} \quad \text { and } \quad r<s<n
$$

We put

$$
m:=n-r \quad \text { and } \quad l:=n-s .
$$

We denote by $H_{n, \mathbf{a}}$ the scheme parametrizing all complete intersections of multi-degree $\mathbf{a}$ in $\mathbb{P}^{n}$. For a point $t$ of $H_{n, \mathbf{a}}$, we denote by $X_{t}$ the corresponding complete intersection. Let $S_{n, \mathbf{a}}$ denote the Zariski closed subset of $H_{n, \mathbf{a}}$ parametrizing all singular complete intersections. It is well-known that $S_{n, \mathbf{a}}$ is an irreducible hypersurface of $H_{n, \mathbf{a}}$, and that, if $u$ is a general point of $S_{n, \mathbf{a}}$, then $X_{u}$ has only one singular point $p$. For $t \in H_{n, \mathbf{a}}$, we denote by $F_{\mathbf{b}}\left(X_{t}\right)$ the scheme parametrizing all complete intersections of multi-degree $\mathbf{b}$ in $\mathbb{P}^{n}$ that are contained in $X_{t}$ as subschemes. If $m>2 l$ and $F_{\mathbf{b}}\left(X_{t}\right) \neq \emptyset$, then we have 
the cylinder homomorphism

$$
\psi_{\mathbf{b}}\left(X_{t}\right): H_{m-2 l}\left(F_{\mathbf{b}}\left(X_{t}\right), \mathbb{Z}\right) \rightarrow H_{m}\left(X_{t}, \mathbb{Z}\right) .
$$

We put $t_{a}:=\operatorname{Card}\left\{i \mid a_{i}=a_{r}\right\}$ and $t_{b}:=\operatorname{Card}\left\{j \mid b_{j}=a_{r}\right\}$.

MAIN THEOREM. Suppose that the following inequalities are satisfied:

$$
\begin{gathered}
a_{i} \geq b_{i} \quad(i=1, \ldots, r), \quad a_{r} \geq b_{s} \\
m-2 l \geq t_{b}-t_{a}, \quad m>2 l
\end{gathered}
$$

Suppose also that, for a general point $u$ of $S_{n, \mathbf{a}}$, there exists a complete intersection of multi-degree $\mathbf{b}$ in $\mathbb{P}^{n}$ that is contained in $X_{u}$, passing through the unique singular point $p$ of $X_{u}$, and smooth at $p$. Then, for a general point $t$ of $H_{n, \mathbf{a}}$, the scheme $F_{\mathbf{b}}\left(X_{t}\right)$ is non-empty, and the image of the cylinder homomorphism $\psi_{\mathbf{b}}\left(X_{t}\right)$ contains the module of vanishing cycles $V_{m}\left(X_{t}, \mathbb{Z}\right)$.

REMARK 2.1. In Remark 4.12 and Proposition 4.15, we will give several conditions equivalent to the second condition of Main Theorem. One of them can be easily tested by means of Gröbner bases, as will be explained in Section 6 .

3. Vanishing cycles and a cylinder homomorphism. In this section, we work in the category of complex analytic spaces and holomorphic maps. We study in general setting the problem when the image of a cylinder homomorphism contains a given vanishing cycle. For the detail of the classical theory of vanishing cycles, we refer to [9].

Let $\varphi: Y \rightarrow \Delta$ be a proper surjective holomorphic map from a smooth irreducible complex analytic space of dimension $m+1 \geq 2$ to the open unit disk $\Delta \subset \mathbb{C}$. For a point $a \in \Delta$, we denote by $Y_{a}$ the fiber $\varphi^{-1}(a)$. Suppose that $\varphi$ has only one critical point $p$, that $p$ is on the central fiber $Y_{0}$, and that the Hessian

$$
H: T_{p} Y \times T_{p} Y \rightarrow \mathbb{C}
$$

of $\varphi$ at $p$ is non-degenerate. We put

$$
\Delta^{\times}:=\Delta \backslash\{0\} .
$$

For any $\varepsilon \in \Delta^{\times}$, the kernel of the homomorphism $H_{m}\left(Y_{\varepsilon}, \mathbb{Z}\right) \rightarrow H_{m}(Y, \mathbb{Z})$ induced from the inclusion $Y_{\varepsilon} \hookrightarrow Y$ is generated by the vanishing cycle $\left[\Sigma_{\varepsilon}\right] \in H_{m}\left(Y_{\varepsilon}, \mathbb{Z}\right)$ associated to the non-degenerate critical point $p$ of $\varphi$.

Let $\varrho: F \rightarrow \Delta$ be a surjective holomorphic map from a smooth irreducible complex analytic space $F$ of dimension $k$ to the unit disk, and let $W$ be a reduced closed analytic subspace of $Y \times_{\Delta} F$ such that the projection $\varpi: W \rightarrow F$ is flat of relative dimension $l>0$. Since $\varphi$ is proper, so is $\varpi$. Let $\gamma: W \rightarrow Y$ be the projection onto the first factor. We obtain the following commutative diagram:

$$
\begin{array}{cr}
W \stackrel{\gamma}{\longrightarrow} Y \\
\varpi \downarrow & \downarrow \varphi \\
F \underset{\varrho}{\longrightarrow} \Delta .
\end{array}
$$


For $u \in F$, the fiber $\varpi^{-1}(u)$ can be regarded as a closed $l$-dimensional analytic subspace of $Y_{\varrho(u)}$ by $\gamma$. For $a \in \Delta$, we put

$$
F_{a}:=\varrho^{-1}(a) \text { and } W_{a}:=\varpi^{-1}\left(F_{a}\right) .
$$

Then we obtain a family of $l$-dimensional closed analytic subspaces of $Y_{a}$ :

$$
\begin{gathered}
W_{a} \longrightarrow Y_{a} \\
\varpi_{a} \downarrow \\
F_{a} .
\end{gathered}
$$

Since the restriction $\varpi_{a}: W_{a} \rightarrow F_{a}$ of $\varpi$ to $W_{a}$ is proper and flat of relative dimension $l$, we have the cylinder homomorphism

$$
\psi_{a}: H_{m-2 l}\left(F_{a}, \mathbb{Z}\right) \rightarrow H_{m}\left(Y_{a}, \mathbb{Z}\right)
$$

associated with the family (3.2) for any $a \in \Delta$.

THEOREM 3.1. We assume $m>2 l>0$.

(1) Suppose that there exists a point $q$ of $W_{0}$ such that $\gamma(q)$ is the critical point $p$ of $\varphi$, that $\varpi$ is smooth at $q$, and that $\gamma$ is an immersion at $q$. Then $k=\operatorname{dim} F$ is less than or equal to $m-2 l+1$.

(2) Suppose moreover that $k=m-2 l+1$. Then $\varpi(q)$ is a critical point of $\varrho$, and the Hessian of $\varrho$ at $\varpi(q)$ is non-degenerate. Let $\varepsilon$ be a point of $\Delta^{\times}$with $|\varepsilon|$ small enough, and let $\left[\sigma_{\varepsilon}\right] \in H_{m-2 l}\left(F_{\varepsilon}, \mathbb{Z}\right)$ be the vanishing cycle associated to the non-degenerate critical point $\varpi(q)$ of $\varrho$. If the vanishing cycle $\left[\Sigma_{\varepsilon}\right] \in H_{m}\left(Y_{\varepsilon}, \mathbb{Z}\right)$ is not a torsion element, then $\psi_{\varepsilon}\left(\left[\sigma_{\varepsilon}\right]\right)$ is equal to $\left[\Sigma_{\varepsilon}\right]$ up to sign.

Proof. (1) Let $U_{W, q}$ be a small open connected neighborhood of $q$ in $W$. We can assume that $\varpi$ is smooth at every point of $U_{W, q}$, and that $\gamma$ embeds $U_{W, q}$ into $Y$. We put

$$
o:=\varpi(q) \text { and } Z:=\varpi^{-1}(o) .
$$

Then $\gamma\left(U_{W, q} \cap Z\right)$ and $\gamma\left(U_{W, q}\right)$ are smooth locally closed analytic subsets of $Y$ passing through $p$. Let $T_{1}$ and $T_{2}$ be the Zariski tangent spaces to $\gamma\left(U_{W, q} \cap Z\right)$ and $\gamma\left(U_{W, q}\right)$ at $p$, respectively. We have $T_{1} \subseteq T_{2} \subseteq T_{p} Y$ and $\operatorname{dim} T_{1}=l, \operatorname{dim} T_{2}=k+l$. We will show that $T_{1}$ and $T_{2}$ are orthogonal with respect to the Hessian $H$ of $\varphi$ at $p$. Let $v$ be an arbitrary vector of $T_{1}$. Since the structure $\varpi \mid U_{W, q}: U_{W, q} \rightarrow F$ of the smooth fibration on $U_{W, q}$ is carried over to $\gamma\left(U_{W, q}\right)$, there exists a holomorphic vector field $\tilde{v}$ defined in a small open neighborhood $U_{Y, p}$ of $p$ in $Y$ such that $\tilde{v}_{p}$ is equal to $v$, and that, if $q^{\prime} \in U_{W, q}$ satisfies $\gamma\left(q^{\prime}\right) \in U_{Y, p}$, then $\tilde{v}_{\gamma\left(q^{\prime}\right)}$ is tangent to the smooth locally closed analytic subset $\gamma\left(U_{W, q} \cap \varpi^{-1}\left(\varpi\left(q^{\prime}\right)\right)\right)$ of $Y$. Since the diagram (3.1) is commutative, the function $\varphi$ is constant on $\gamma\left(\varpi^{-1}\left(\varpi\left(q^{\prime}\right)\right)\right)$ for any $q^{\prime} \in U_{W, q}$, and hence the holomorphic function $\tilde{v}(\varphi)$ is constantly zero on $\gamma\left(U_{W, q}\right) \cap U_{Y, p}$, which means that the following holds for any $w \in T_{2}$ :

$$
H(w, v):=w(\tilde{v}(\varphi))=0 .
$$

Thus $T_{1}$ is contained in the orthogonal complement $T_{2}^{\perp}$ of $T_{2}$ with respect to $H$. Since $H$ is non-degenerate, we have

$$
l=\operatorname{dim} T_{1} \leq \operatorname{dim} T_{p} Y-\operatorname{dim} T_{2}=(m+1)-(k+l) .
$$


Therefore we obtain $k \leq m+1-2 l$.

(2) From now on, we assume $k=m+1-2 l$. Then we have $T_{1}=T_{2}^{\perp}$. Hence $H$ induces a non-degenerate symmetric bilinear form

$$
H^{\prime}: T_{2} / T_{1} \times T_{2} / T_{1} \rightarrow \mathbb{C}
$$

Since $\varpi$ is smooth at $q$, there is a local holomorphic section $s: U_{F, o} \rightarrow W$ of $\varpi$ defined in a small open neighborhood $U_{F, o}$ of $o=\varpi(q)$ in $F$ such that $s(o)=q$. We take $U_{F, o}$ so small that $s\left(U_{F, o}\right) \subset U_{W, q}$ holds. Let $S$ be the image of $\gamma \circ s$, which is a smooth locally closed analytic subset of $Y$ passing through $p$, and let $T_{3}$ be the Zariski tangent space to $S$ at $p$. We have $T_{2}=T_{1} \oplus T_{3}$. It follows from the non-degeneracy of $H^{\prime}$ that the restriction $H \mid T_{3}: T_{3} \times T_{3} \rightarrow \mathbb{C}$ of $H$ to $T_{3}$ is also non-degenerate. Since $\gamma \circ s$ yields an isomorphism from $U_{F, o}$ to $S$, and $\varrho$ coincides on $U_{F, o}$ with

$$
U_{F, o} \stackrel{\gamma \circ s}{\longrightarrow} S \stackrel{\varphi \mid S}{\longrightarrow} \Delta,
$$

the point $o$ is a critical point of $\varrho$. Moreover, the Hessian of $\varrho$ at $o$ is equal to $H \mid T_{3}$ via the isomorphism $(d(\gamma \circ s))_{o}: T_{o} F \stackrel{\sim}{\rightarrow} T_{3}$, and hence is non-degenerate.

We will describe the holomorphic maps in the diagram (3.1) in terms of local coordinates. Let $t$ be the coordinate on $\Delta$. There exist local analytic coordinates $x=$ $\left(x_{1}, \ldots, x_{k}\right)$ on $F$ with the center $o$ such that $\varrho$ is given by

$$
\varrho^{*} t=x_{1}^{2}+\ldots+x_{k}^{2} .
$$

Since $\varpi$ is smooth at $q$, there exists a local analytic coordinate system

$$
\left(w, w^{\prime}\right)=\left(w_{1}, \ldots, w_{k}, w_{1}^{\prime}, \ldots, w_{l}^{\prime}\right)
$$

on $W$ with the center $q$ such that $\varpi$ is given by

$$
\varpi^{*} x_{i}=w_{i} \quad(i=1, \ldots, k) .
$$

Since $\gamma$ is an immersion at $q$, there exist local analytic coordinates

$$
\left(y, y^{\prime}, y^{\prime \prime}\right)=\left(y_{1}, \ldots, y_{k}, y_{1}^{\prime}, \ldots, y_{l}^{\prime}, y_{1}^{\prime \prime}, \ldots, y_{l}^{\prime \prime}\right)
$$

on $Y$ with the center $p$ such that $\gamma$ is given by

$$
\begin{cases}\gamma^{*} y_{i}=w_{i} & (i=1, \ldots, k), \\ \gamma^{*} y_{j}^{\prime}=w_{j}^{\prime} & (j=1, \ldots, l), \\ \gamma^{*} y_{j}^{\prime \prime}=0 & (j=1, \ldots, l) .\end{cases}
$$

(Note that $\operatorname{dim} Y$ is equal to $m+1=k+2 l$.) Then the locally closed analytic subset $\gamma\left(U_{W, q}\right)$ of $Y$ is defined by $y_{1}^{\prime \prime}=\ldots=y_{l}^{\prime \prime}=0$ locally around $p$. From the commutativity of the diagram (3.1), it follows that $\varphi^{*} t$ and $y_{1}^{2}+\ldots+y_{k}^{2}$ coincide on $\gamma\left(U_{W, q}\right)$. Therefore, in a small neighborhood of $p$, the function $\varphi^{*} t$ is written as follows:

$$
y_{1}^{2}+\ldots+y_{k}^{2}+a_{1} y_{1}^{\prime \prime}+\ldots+a_{l} y_{l}^{\prime \prime},
$$

where $a=\left(a_{1}, \ldots, a_{l}\right)$ is a system of holomorphic functions defined locally around $p$. Since $p$ is a critical point of $\varphi$, we have $a_{1}(p)=\ldots=a_{l}(p)=0$. The non-degeneracy of 
the Hessian $H$ of $\varphi$ at $p$ implies that the $l \times l$ matrix

$$
\left(\frac{\partial a_{i}}{\partial y_{j}^{\prime}}(p)\right)_{i, j=1, \ldots, l}
$$

is non-degenerate. Hence $\left(y, a, y^{\prime \prime}\right)$ is another local analytic coordinate system on $Y$ with the center $p$. We replace $y^{\prime}$ with $a$. Then we have

$$
\varphi^{*} t=y_{1}^{2}+\ldots+y_{k}^{2}+y_{1}^{\prime} y_{1}^{\prime \prime}+\ldots+y_{l}^{\prime} y_{l}^{\prime \prime} .
$$

We can make coordinate transformation on $w^{\prime}$ according to the coordinate transformation on $y^{\prime}$ so that $(3.5)$ remains valid. We put

$$
\begin{cases}z_{i}:=y_{i} & (i=1, \ldots, k), \\ z_{k+j}:=\left(y_{j}^{\prime}+y_{j}^{\prime \prime}\right) / 2 & (j=1, \ldots, l), \\ z_{k+l+j}:=\sqrt{-1}\left(y_{j}^{\prime}-y_{j}^{\prime \prime}\right) / 2 & (j=1, \ldots, l) .\end{cases}
$$

Then we have

$$
\varphi^{*} t=z_{1}^{2}+\ldots+z_{m+1}^{2} .
$$

Let $\eta$ be a sufficiently small positive real number, and let $B_{\eta}$ be the closed ball in $Y$ defined by

$$
\left|z_{1}\right|^{2}+\ldots+\left|z_{m+1}\right|^{2} \leq \eta
$$

Let $\varepsilon$ be a positive real number that is small enough compared with $\eta$. Let $s$ be a real number satisfying $0<s \leq \varepsilon$. The closed subset

$$
Y_{s} \cap B_{\eta}=\left\{\left.\left(z_{1}, \ldots, z_{m+1}\right)|| z_{1}\right|^{2}+\ldots+\left|z_{m+1}\right|^{2} \leq \eta, z_{1}^{2}+\ldots+z_{m+1}^{2}=s\right\}
$$

of $Y_{s}=\varphi^{-1}(s)$ is homeomorphic to the total space

$$
E:=\left\{(u, v) \in \mathbb{R}^{m+1} \times \mathbb{R}^{m+1} \mid\|u\|=1,\|v\| \leq 1, u \perp v\right\}
$$

of the unit disk tangent bundle $\tau: E \rightarrow S^{m}$ of the $m$-dimensional sphere

$$
S^{m}:=\left\{u \in \mathbb{R}^{m+1} \mid\|u\|=1\right\},
$$

where the projection $\tau$ is given by $\tau(u, v)=u$. We identify $S^{m}$ with the zero section of $\tau: E \rightarrow S^{m}$. The homeomorphism $h_{s}: Y_{s} \cap B_{\eta} \stackrel{\sim}{\rightarrow} E$ is written explicitly as follows:

$$
u=\frac{\operatorname{Re}(z)}{\|\operatorname{Re}(z)\|}, \quad v=\sqrt{\frac{2}{\eta-s}} \operatorname{Im}(z) .
$$

Its inverse $h_{s}^{-1}: E \simeq Y_{s} \cap B_{\eta}$ is given by the following:

$$
z=\sqrt{s+\left(\frac{\eta-s}{2}\right)\|v\|^{2}} \cdot u+\sqrt{-\left(\frac{\eta-s}{2}\right)} \cdot v .
$$

The sphere $S^{m} \subset E$ is mapped by $h_{s}^{-1}$ to the closed submanifold

$$
\Sigma_{s}:=\left\{\left(z_{1}, \ldots, z_{m+1}\right) \in Y \mid z_{1}^{2}+\ldots+z_{m+1}^{2}=s, \operatorname{Im}\left(z_{i}\right)=0(i=1, \ldots, m+1)\right\}
$$

of $Y_{s}$. We put an orientation on $\Sigma_{s}$. Then this topological $m$-cycle $\Sigma_{s}$ represents the vanishing cycle $\left[\Sigma_{s}\right] \in H_{m}\left(Y_{s}, \mathbb{Z}\right)$, which generates the kernel of the homomorphism $H_{m}\left(Y_{s}, \mathbb{Z}\right) \rightarrow H_{m}(Y, \mathbb{Z})$ induced from $Y_{s} \hookrightarrow Y$. 
For $s \in(0, \varepsilon]$, let $\sigma_{s}$ denote the $(m-2 l)$-dimensional sphere contained in $F_{s}=\varrho^{-1}(s)$ defined by

$$
\sigma_{s}:=\left\{\left(x_{1}, \ldots, x_{k}\right) \in F \mid x_{1}^{2}+\ldots+x_{k}^{2}=s, \operatorname{Im}\left(x_{i}\right)=0(i=1, \ldots, k)\right\} .
$$

With an orientation, this topological $(m-2 l)$-cycle $\sigma_{s}$ represents the vanishing cycle $\left[\sigma_{s}\right] \in H_{m-2 l}\left(F_{s}, \mathbb{Z}\right)$ associated to the non-degenerate critical point $o$ of $\varrho$. Since $\varpi$ is proper and flat of relative dimension $l$, the inverse image $\varpi^{-1}\left(\sigma_{s}\right)$ of the oriented sphere $\sigma_{s}$ can be considered as a topological $m$-cycle in $W_{s}=\varpi^{-1}\left(F_{s}\right)$. The image $\psi_{\varepsilon}\left(\left[\sigma_{\varepsilon}\right]\right)$ of $\left[\sigma_{\varepsilon}\right] \in H_{m-2 l}\left(F_{\varepsilon}, \mathbb{Z}\right)$ by the cylinder homomorphism $\psi_{\varepsilon}: H_{m-2 l}\left(F_{\varepsilon}, \mathbb{Z}\right) \rightarrow H_{m}\left(Y_{\varepsilon}, \mathbb{Z}\right)$ is represented by the topological $m$-cycle

$$
\gamma \mid \varpi^{-1}\left(\sigma_{\varepsilon}\right): \varpi^{-1}\left(\sigma_{\varepsilon}\right) \rightarrow Y_{\varepsilon}
$$

Since the sphere $\sigma_{\varepsilon}$ bounds an $(m-2 l+1)$-dimensional closed ball in $F$, the topological $m$-cycle $\gamma \mid \varpi^{-1}\left(\sigma_{\varepsilon}\right)$ is a boundary of a topological $(m+1)$-chain in $Y$; that is, $\psi_{\varepsilon}\left(\left[\sigma_{\varepsilon}\right]\right)$ belongs to the kernel of $H_{m}\left(Y_{\varepsilon}, \mathbb{Z}\right) \rightarrow H_{m}(Y, \mathbb{Z})$. Hence there exists an integer $c$ such that the following holds in $H_{m}\left(Y_{\varepsilon}, \mathbb{Z}\right)$ :

$$
\psi_{\varepsilon}\left(\left[\sigma_{\varepsilon}\right]\right)=c\left[\Sigma_{\varepsilon}\right]
$$

We will show that, if $\left[\Sigma_{\varepsilon}\right]$ is not a torsion element in $H_{m}\left(Y_{\varepsilon}, \mathbb{Z}\right)$, then $c$ is \pm 1 .

We put

$$
Y_{[0, \varepsilon]}:=\varphi^{-1}([0, \varepsilon])=\bigcup_{s \in[0, \varepsilon]} Y_{s} .
$$

For any closed subset $A$ of $Y_{[0, \varepsilon]}$, we set

$$
A^{\sharp}:=A \backslash\left(A \cap B_{\eta}^{\circ}\right), \quad A^{b}:=A \cap B_{\eta} \quad \text { and } \quad \partial^{B} A:=A \cap \partial B_{\eta},
$$

where $B_{\eta}^{\circ}$ is the interior of the closed ball $B_{\eta}$, and $\partial B_{\eta}$ is the boundary of $B_{\eta}$. The sharp $\sharp$ means "outside the ball", and the flat $b$ means "inside the ball". The explicit descriptions (3.9) and (3.10) of the homeomorphism $h_{s}: Y_{s}^{\mathrm{b}} \stackrel{\sim}{\rightarrow}$ for $s \in(0, \varepsilon]$ show that the restriction $h_{s} \mid \partial^{B} Y_{s}: \partial^{B} Y_{s} \sim \partial E$ of $h_{s}$ to $\partial^{B} Y_{s}$ can be extended to a homeomorphism from

$$
\partial^{B} Y_{0}=\left\{\left.\left(z_{1}, \ldots, z_{m+1}\right)|| z_{1}\right|^{2}+\ldots+\left|z_{m+1}\right|^{2}=\eta, z_{1}^{2}+\ldots+z_{m+1}^{2}=0\right\}
$$

to $\partial E=\{(u, v) \in E \mid\|v\|=1\}$ smoothly. We denote these homeomorphisms by

$$
\partial^{B} h_{s}: \partial^{B} Y_{s} \stackrel{\sim}{\rightarrow} \partial E \quad(s \in[0, \varepsilon]) .
$$

The homeomorphism $\partial^{B} h_{0}: \partial^{B} Y_{0} \simeq \partial E$ is given by:

$$
u=\sqrt{\frac{2}{\eta}} \operatorname{Re}(z), \quad v=\sqrt{\frac{2}{\eta}} \operatorname{Im}(z), \quad \text { and } \quad z=\sqrt{\frac{\eta}{2}}(u+\sqrt{-1} v) .
$$

Putting these homeomorphisms $\partial^{B} h_{s}(s \in[0, \varepsilon])$ together, we obtain a trivialization

$$
\partial^{B} h: \partial^{B} Y_{[0, \varepsilon]} \stackrel{\sim}{\rightarrow} \partial E \times[0, \varepsilon]
$$

of the restriction $\varphi \mid \partial^{B} Y_{[0, \varepsilon]}: \partial^{B} Y_{[0, \varepsilon]} \rightarrow[0, \varepsilon]$ of $\varphi$ to $\partial^{B} Y_{[0, \varepsilon]}$ over $[0, \varepsilon]$. Let

$$
\partial^{B} f: \partial^{B} Y_{[0, \varepsilon]} \stackrel{\sim}{\longrightarrow} \partial^{B} Y_{\varepsilon} \times[0, \varepsilon]
$$


be the trivialization of $\varphi \mid \partial^{B} Y_{[0, \varepsilon]}$ obtained by composing $\partial^{B} h$ and $\left(\partial^{B} h_{\varepsilon} \times \text { id }\right)^{-1}$. Since the only critical point $p$ of $\varphi$ is not contained in $Y_{[0, \varepsilon]}^{\sharp}$, we can show by Ehresmann's fibration theorem for the manifolds with boundaries that the trivialization $\partial^{B} f$ extends to a trivialization

$$
\left(f^{\sharp}, \partial^{B} f\right):\left(Y_{[0, \varepsilon]}^{\sharp}, \partial^{B} Y_{[0, \varepsilon]}\right) \stackrel{\sim}{\rightarrow}\left(Y_{\varepsilon}^{\sharp}, \partial^{B} Y_{\varepsilon}\right) \times[0, \varepsilon]
$$

of $\varphi \mid Y_{[0, \varepsilon]}^{\sharp}: Y_{[0, \varepsilon]}^{\sharp} \rightarrow[0, \varepsilon]$ in such a way that the restriction of $\left(f^{\sharp}, \partial^{B} f\right)$ to the fiber over $\varepsilon$ is the identity map. For $s \in[0, \varepsilon]$, let

$$
\left(f_{s}^{\sharp}, \partial^{B} f_{s}\right):\left(Y_{s}^{\sharp}, \partial^{B} Y_{s}\right) \stackrel{\sim}{\rightarrow}\left(Y_{\varepsilon}^{\sharp}, \partial^{B} Y_{\varepsilon}\right)
$$

denote the restriction of $\left(f^{\sharp}, \partial^{B} f\right)$ to the fiber over $s$.

We put

$$
C_{s}:=\gamma\left(\varpi^{-1}\left(\sigma_{s}\right)\right) \subset Y_{s}
$$

When $s$ approaches 0 , this closed subset $C_{s}$ degenerates into $C_{0}:=\gamma\left(\varpi^{-1}(o)\right)$, which is an $l$-dimensional closed analytic subset of $Y_{0}$. We decompose $\varpi^{-1}\left(\sigma_{s}\right)$ into the union of $\varpi^{-1}\left(\sigma_{s}\right)^{(\sharp)}$ and $\varpi^{-1}\left(\sigma_{s}\right)^{(b)}$, where

$$
\begin{aligned}
& \varpi^{-1}\left(\sigma_{s}\right)^{(\sharp)}:=\varpi^{-1}\left(\sigma_{s}\right) \backslash\left(\gamma^{-1}\left(B_{\eta}^{\circ}\right) \cap \varpi^{-1}\left(\sigma_{s}\right)\right), \\
& \varpi^{-1}\left(\sigma_{s}\right)^{(b)}:=\gamma^{-1}\left(B_{\eta}\right) \cap \varpi^{-1}\left(\sigma_{s}\right) .
\end{aligned}
$$

Since $\eta$ and $\varepsilon$ are small enough, and $W$ is a subspace of $Y \times F$, we have

$$
\varpi^{-1}\left(\sigma_{s}\right)^{(b)}=W \cap\left(B_{\eta} \times \sigma_{s}\right) \subset U_{W, q}
$$

for all $s \in[0, \varepsilon]$, where $U_{W, q}$ is the open neighborhood of $q$ in $W$ that was introduced at the beginning of the proof. Recalling that $\gamma$ embeds $U_{W, q}$ into $Y$, we see that the map $\gamma$ yields a homeomorphism from $\varpi^{-1}\left(\sigma_{\varepsilon}\right)^{(b)}$ to $C_{\varepsilon}^{b}$. By definition, $\gamma$ maps $\varpi^{-1}\left(\sigma_{\varepsilon}\right)^{(\sharp)}$ to $C_{\varepsilon}^{\sharp}$. We then define a closed subset $\widetilde{C}_{\varepsilon}$ of $Y_{\varepsilon}$ by

$$
\widetilde{C}_{\varepsilon}:=C_{\varepsilon}^{b} \cup\left(\bigcup_{s \in[0, \varepsilon]} \partial^{B} f_{s}\left(\partial^{B} C_{s}\right)\right) \cup f_{0}^{\sharp}\left(C_{0}^{\sharp}\right) .
$$

Note that we have

$$
\partial^{B} \widetilde{C}_{\varepsilon}=\bigcup_{s \in[0, \varepsilon]} \partial^{B} f_{s}\left(\partial^{B} C_{s}\right) \quad \text { and } \quad \widetilde{C}_{\varepsilon}^{b}=C_{\varepsilon}^{b} \cup \partial^{B} \widetilde{C}_{\varepsilon}, \quad \widetilde{C}_{\varepsilon}^{\sharp}=\partial^{B} \widetilde{C}_{\varepsilon} \cup f_{0}^{\sharp}\left(C_{0}^{\sharp}\right) .
$$

Using the trivialization $\left(f^{\sharp}, \partial^{B} f\right)$, we can "squeeze" the topological $m$-cycle

$$
\gamma \mid \varpi^{-1}\left(\sigma_{\varepsilon}\right): \varpi^{-1}\left(\sigma_{\varepsilon}\right) \rightarrow Y_{\varepsilon}
$$

outside the ball so that the image is contained in $\widetilde{C}_{\varepsilon}$. More precisely, we can construct a homotopy from $\gamma \mid \varpi^{-1}\left(\sigma_{\varepsilon}\right): \varpi^{-1}\left(\sigma_{\varepsilon}\right) \rightarrow Y_{\varepsilon}$ to a continuous map $\beta: \varpi^{-1}\left(\sigma_{\varepsilon}\right) \rightarrow Y_{\varepsilon}$ with the following properties:

$(\beta-1)$ The image $\beta\left(\varpi^{-1}\left(\sigma_{\varepsilon}\right)\right)$ of $\beta$ coincides with $\widetilde{C}_{\varepsilon}$.

$(\beta-2)$ The homotopy is stationary on $\varpi^{-1}\left(\sigma_{\varepsilon}\right)^{(b)}$. In particular, $\beta$ yields a homeomorphism from $\varpi^{-1}\left(\sigma_{\varepsilon}\right)^{(b)}$ to the first piece $C_{\varepsilon}^{b}$ of the decomposition (3.14).

$(\beta-3)$ The image $\beta\left(\varpi^{-1}\left(\sigma_{\varepsilon}\right)^{(\sharp)}\right)$ of $\varpi^{-1}\left(\sigma_{\varepsilon}\right)^{(\sharp)}$ by $\beta$ is contained in $\widetilde{C}_{\varepsilon}^{\sharp}$. 
(See Figure 1.) Since $\psi_{\varepsilon}\left(\left[\sigma_{\varepsilon}\right]\right)$ is represented by $\gamma \mid \varpi^{-1}\left(\sigma_{\varepsilon}\right)$, it is also represented by the topological $m$-cycle $\beta$.

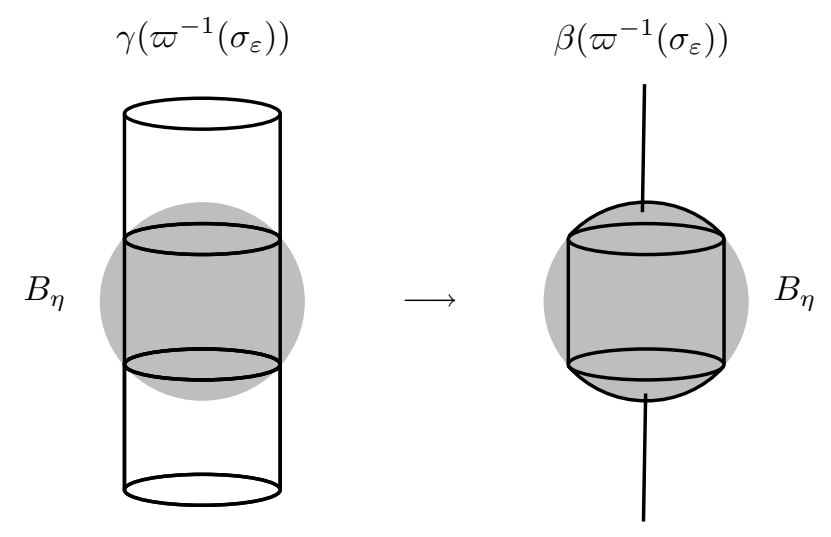

Figure 1. Homotopy from $\gamma \mid \varpi^{-1}\left(\sigma_{\varepsilon}\right)$ to $\beta$

From $(3.13)$ and $(3.3),(3.4),(3.5),(3.7)$, we see that $C_{s}^{b}(s \in[0, \varepsilon])$ is given in terms of the local coordinate system $z$ by

$$
\left\{\begin{array}{l}
\left|z_{1}\right|^{2}+\ldots+\left|z_{m+1}\right|^{2} \leq \eta \\
z_{1}^{2}+\ldots+z_{k}^{2}=s \\
\operatorname{Im}\left(z_{i}\right)=0 \quad(i=1, \ldots, k), \\
y_{j}^{\prime \prime}=z_{k+j}+\sqrt{-1} z_{k+l+j}=0 \quad(j=1, \ldots, l) .
\end{array}\right.
$$

For $s \in[0, \varepsilon]$, let $G_{s}$ be the closed subset of $E$ defined by the following equations:

$$
\left\{\begin{array}{l}
\left(2 s+(\eta-s)\|v\|^{2}\right)\left(u_{1}^{2}+\ldots+u_{k}^{2}\right)=2 s, \\
v_{1}=\ldots=v_{k}=0 \\
v_{k+j}=-g_{s}(\|v\|) \cdot u_{k+l+j} \quad(j=1, \ldots, l), \\
v_{k+l+j}=g_{s}(\|v\|) \cdot u_{k+j} \quad(j=1, \ldots, l),
\end{array}\right.
$$

where

$$
g_{s}(\|v\|):=\sqrt{\frac{2 s}{\eta-s}+\|v\|^{2}} .
$$

Then, for $s \in(0, \varepsilon]$, the homeomorphism $h_{s}: Y_{s}^{b} \stackrel{\sim}{\rightarrow} E$ maps $C_{s}^{b}$ to $G_{s}$. In particular, the first piece $C_{\varepsilon}^{b}$ of the decomposition (3.14) of $\widetilde{C}_{\varepsilon}$ is mapped homeomorphically to $G_{\varepsilon}$ by $h_{\varepsilon}$. It is easy to check that, for any $s \in[0, \varepsilon]$ (including $s=0$ ), the homeomorphism $\partial^{B} h_{s}: \partial^{B} Y_{s} \stackrel{\sim}{\rightarrow} \partial E$ maps $\partial^{B} C_{s}$ to $G_{s} \cap \partial E$. We put

$$
T_{\varepsilon}:=\left\{u \in S^{m} \mid u_{1}^{2}+\ldots+u_{k}^{2}<2 \varepsilon /(\eta+\varepsilon)\right\},
$$

and let $T_{\varepsilon}^{-}$be the closure of $T_{\varepsilon}$. We can easily check that the projection $\tau: E \rightarrow S^{m}$ induces a homeomorphism from $G_{\varepsilon}$ to $S^{m} \backslash T_{\varepsilon}$, and that, for any $s \in[0, \varepsilon], G_{s} \cap \partial E$ is contained in $\tau^{-1}\left(T_{\varepsilon}^{-}\right) \cap \partial E$. In particular, the second piece $\partial^{B} \widetilde{C}_{\varepsilon}$ of the decomposition (3.14) is mapped by $\partial^{B} h_{\varepsilon}$ into $\tau^{-1}\left(T_{\varepsilon}^{-}\right) \cap \partial E$. 
Let $a$ be a point of $S^{m} \backslash T_{\varepsilon}^{-}$. Then the closed subset $h_{\varepsilon}^{-1}\left(\tau^{-1}(a)\right)$ of $Y_{\varepsilon}^{b}$ intersects the first piece $C_{\varepsilon}^{b}$ of the decomposition (3.14) at only one point, which is in the interior of $C_{\varepsilon}^{b}$, and the intersection is transverse. Moreover, $h_{\varepsilon}^{-1}\left(\tau^{-1}(a)\right)$ is disjoint from the second piece $\partial^{B} \widetilde{C}_{\varepsilon}$ of the decomposition (3.14). The third piece $f_{0}^{\sharp}\left(C_{0}^{\sharp}\right)$ is a topological $2 l$-cycle in $\left(Y_{\varepsilon}^{\sharp}, \partial^{B} Y_{\varepsilon}\right)$, because $C_{0}$ is a topological $2 l$-cycle in $Y_{0}$.

If $\left[\Sigma_{\varepsilon}\right] \in H_{m}\left(Y_{\varepsilon}, \mathbb{Z}\right)$ is zero, then $\psi_{\varepsilon}\left(\left[\sigma_{\varepsilon}\right]\right)=0$ by $(3.11)$ and hence there is nothing to prove. Suppose that $\left[\Sigma_{\varepsilon}\right]$ is not zero and not a torsion element. Then there exists a homology class $[\Theta] \in H_{m}\left(Y_{\varepsilon}, \mathbb{Z}\right)$ such that the intersection number $\left[\Sigma_{\varepsilon}\right] \cdot[\Theta]$ of $\left[\Sigma_{\varepsilon}\right]$ and $[\Theta]$ in $Y_{\varepsilon}$ is not zero. In order to show that the integer $c$ in $(3.11)$ is \pm 1 , it is enough to prove that

$$
\psi_{\varepsilon}\left(\left[\sigma_{\varepsilon}\right]\right) \cdot[\Theta]= \pm\left[\Sigma_{\varepsilon}\right] \cdot[\Theta]
$$

Multiplying $[\Theta]$ by a positive integer if necessary, we can assume that $[\Theta]$ is represented by a compact oriented $m$-dimensional differentiable submanifold $\Theta$ of $Y_{\varepsilon}([20])$. By the elementary transversality theorem (see, for example, [7]), we can move $\Theta$ in $Y_{\varepsilon}$ in such a way that the following hold:

$(\Theta-1)$ The closed subset $h_{\varepsilon}\left(\Theta^{b}\right)$ of $E$ is a union of finite number of fibers of $\tau: E \rightarrow S^{m}$ over points in $S^{m} \backslash T_{\varepsilon}^{-}$.

$\left(\Theta\right.$-2) The topological $m$-cycle $\Theta^{\sharp}$ of $\left(Y_{\varepsilon}^{\sharp}, \partial^{B} Y_{\varepsilon}\right)$ is disjoint from the topological $2 l$-cycle $f_{0}^{\sharp}\left(C_{0}^{\sharp}\right)$. Here we use the assumption $m>2 l$.

From $(\Theta-1)$ and $(\Theta-2)$, the points $\Theta \cap \widetilde{C}_{\varepsilon}$ are contained in the interior of the first piece $C_{\varepsilon}^{b}$ of the decomposition (3.14) of $\widetilde{C}_{\varepsilon}$, and the intersections are all transverse. Moreover, the total intersection number of $\Theta$ and $\widetilde{C}_{\varepsilon}$ is equal to that of $\Theta$ and $\Sigma_{\varepsilon}$ up to sign, because both of them are equal, up to sign, to the number of fibers of $\tau$ constituting $h_{\varepsilon}\left(\Theta^{b}\right)$ (counted with signs according to the orientation). Combining these with the properties $(\beta-1)-(\beta-3)$ of the topological $m$-cycle $\beta$, we see that $[\beta] \cdot[\Theta]= \pm\left[\Sigma_{\varepsilon}\right] \cdot[\Theta]$. We have seen that $\psi_{\varepsilon}\left(\left[\sigma_{\varepsilon}\right]\right)$ is represented by $\beta$. Thus we obtain $(3.15)$.

4. The universal family. In this section, we will construct the universal family of the incidence varieties of complete intersections in a complex projective space $\mathbb{P}^{n}$.

First we fix some notation. Let

$$
R=\bigoplus_{d=0}^{\infty} R_{d}:=\mathbb{C}\left[x_{0}, \ldots, x_{n}\right]
$$

be the polynomial ring of $n+1$ variables with coefficients in $\mathbb{C}$ graded by the degree $d$ of polynomials. We set $R_{d}:=0$ for $d<0$. Let $M$ be a graded $R$-module. We denote by $M_{d}$ the vector space consisting of homogeneous elements of $M$ with degree $d$. For an integer $k$, let $M(k)$ be the $R$-module $M$ with grading shifted by

$$
M(k)_{d}:=M_{k+d} .
$$

For another graded $R$-module $N$, let $\operatorname{Hom}(M, N)_{0}$ denote the vector space of degreepreserving homomorphisms from $M$ to $N$. Let $\mathbf{c}=\left(c_{1}, \ldots, c_{t}\right)$ be a sequence of positive 
integers. We assume $t<n$. Let us define the graded free $R$-module $M_{\mathbf{c}}$ by

$$
M_{\mathbf{c}}:=\bigoplus_{i=1}^{t} R\left(c_{i}\right) .
$$

An element of $M_{\mathbf{c}}$ is written as a column vector. Let $f=\left(f_{1}, \ldots, f_{t}\right)^{T}$ be an element of $\left(M_{\mathbf{c}}\right)_{0}=\bigoplus R_{c_{i}}$, where $f_{i}$ is a homogeneous polynomial of degree $c_{i}$. We denote by $J_{f}$ the homogeneous ideal of $R$ generated by $f_{1}, \ldots, f_{t}$. There exists a Zariski open dense subset $\left(M_{\mathbf{c}}\right)_{0}^{c i}$ of the vector space $\left(M_{\mathbf{c}}\right)_{0}$ consisting of all $f \in\left(M_{\mathbf{c}}\right)_{0}$ such that the ideal $J_{f}$ defines a complete intersection of multi-degree $\mathbf{c}$ in $\mathbb{P}^{n}=\operatorname{Proj} R$. For $f \in\left(M_{\mathbf{c}}\right)_{0}^{c i}$, let $Y_{\langle f\rangle}$ denote the complete intersection defined by $J_{f}$. It is well-known that, for any integer $\nu$, the dimension of the vector space

$$
H^{0}\left(Y_{\langle f\rangle}, \mathcal{O}(\nu)\right)=\left(\left(R / J_{f}\right)(\nu)\right)_{0}
$$

is independent of the choice of $f \in\left(M_{\mathbf{c}}\right)_{0}^{c i}$.

Let $H_{n, \mathbf{c}}$ denote the scheme parametrizing all complete intersections of multi-degree c in $\mathbb{P}^{n}$. It is well-known that $H_{n, \mathbf{c}}$ is a smooth irreducible quasi-projective scheme. For an element $f \in\left(M_{\mathbf{c}}\right)_{0}^{c i}$, let $\langle f\rangle$ denote the point of $H_{n, \mathbf{c}}$ corresponding to the complete intersection $Y_{\langle f\rangle}$. We have a surjective morphism

$$
q_{\mathbf{c}}:\left(M_{\mathbf{c}}\right)_{0}^{c i} \rightarrow H_{n, \mathbf{c}}
$$

that maps $f$ to $\langle f\rangle$. Let $\mathcal{Y}_{\mathbf{c}} \subset \mathbb{P}^{n} \times H_{n, \mathbf{c}}$ be the universal family of complete intersections of multi-degree $\mathbf{c}$ in $\mathbb{P}^{n}$ with the diagram of the projections

$$
\begin{aligned}
& \mathcal{Y}_{\mathbf{c}} \stackrel{\tau_{\mathbf{c}}}{\longrightarrow} \mathbb{P}^{n} \\
& \phi_{\mathbf{c}} \downarrow \\
& H_{n, \mathbf{c}} .
\end{aligned}
$$

Proposition 4.1.

(1) The morphism $q_{\mathbf{c}}$ is smooth.

(2) The morphism $\tau_{\mathbf{c}}$ is smooth.

In particular, $\mathcal{Y}_{\mathbf{c}}$ is smooth.

Proof. (1) The Zariski tangent space to $H_{n, \mathbf{c}}$ at $\langle f\rangle$ is given by

$$
T_{\langle f\rangle} H_{n, \mathbf{c}}=H^{0}\left(Y_{\langle f\rangle}, \mathcal{N}_{Y_{\langle f\rangle} / \mathbb{P}^{n}}\right)=\left(M_{\mathbf{c}} / J_{f} M_{\mathbf{c}}\right)_{0},
$$

where $\mathcal{N}_{Y_{\langle f\rangle} / \mathbb{P}^{n}}$ is the normal sheaf of $Y_{\langle f\rangle}$ in $\mathbb{P}^{n}$, which is isomorphic to $\bigoplus_{i=1}^{t} \mathcal{O}\left(c_{i}\right)$. By (4.1) and $T_{f}\left(M_{\mathbf{c}}\right)_{0}^{c i} \cong\left(M_{\mathbf{c}}\right)_{0}$, the linear map $\left(d q_{\mathbf{c}}\right)_{f}: T_{f}\left(M_{\mathbf{c}}\right)_{0}^{c i} \rightarrow T_{\langle f\rangle} H_{n, \mathbf{c}}$ is identified with the quotient homomorphism $\left(M_{\mathbf{c}}\right)_{0} \rightarrow\left(M_{\mathbf{c}} / J_{f} M_{\mathbf{c}}\right)_{0}$. Hence $q_{\mathbf{c}}$ is smooth.

(2) Let $P=(p,\langle f\rangle)$ be a point of $\mathcal{Y}_{\mathbf{c}}$, where $p$ is a point of $Y_{\langle f\rangle}$, and let $I_{p}$ be the homogeneous ideal of $R$ defining the point $p$. The kernel of $\left(d \tau_{\mathbf{c}}\right)_{P}: T_{P} \mathcal{Y}_{\mathbf{c}} \rightarrow T_{p} \mathbb{P}^{n}$ is mapped isomorphically to a subspace of $T_{\langle f\rangle} H_{n, \mathbf{c}}$ by $\left(d \phi_{\mathbf{c}}\right)_{P}: T_{P} \mathcal{Y}_{\mathbf{c}} \rightarrow T_{\langle f\rangle} H_{n, \mathbf{c}}$. This subspace coincides with the subspace $\left(I_{p} M_{\mathbf{c}} / J_{f} M_{\mathbf{c}}\right)_{0}$ of $\left(M_{\mathbf{c}} / J_{f} M_{\mathbf{c}}\right)_{0}$ under the identification (4.1). Since $\operatorname{dim}\left(M_{\mathbf{c}} / I_{p} M_{\mathbf{c}}\right)_{0}=t, \operatorname{dim} \operatorname{Ker}\left(d \tau_{\mathbf{c}}\right)_{P}$ is equal to $\operatorname{dim} H_{n, \mathbf{c}}-t=$ $\operatorname{dim} \mathcal{Y}_{\mathbf{c}}-n$ for any point $P \in \mathcal{Y}_{\mathbf{c}}$. 
Let $\mathbf{a}=\left(a_{1}, \ldots, a_{r}\right)$ and $\mathbf{b}=\left(b_{1}, \ldots, b_{s}\right)$ be two sequences of integers satisfying condition (2.1). Instead of $\mathcal{Y}_{\mathbf{a}}$ and $\mathcal{Y}_{\mathbf{b}}$, we denote by

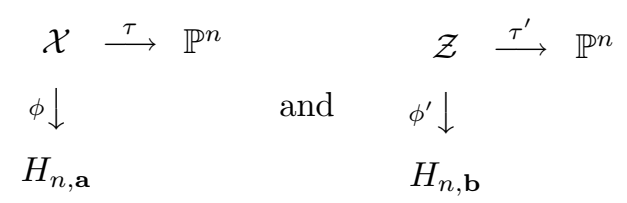

the universal families over $H_{n, \mathbf{a}}$ and $H_{n, \mathbf{b}}$. For $f \in\left(M_{\mathbf{a}}\right)_{0}^{c i}$ and $g \in\left(M_{\mathbf{b}}\right)_{0}^{c i}$, we denote by $X_{\langle f\rangle}$ and $Z_{\langle g\rangle}$ the complete intersections corresponding to $\langle f\rangle \in H_{n, \mathbf{a}}$ and $\langle g\rangle \in H_{n, \mathbf{b}}$, respectively.

An element $h$ of $\operatorname{Hom}\left(M_{\mathbf{b}}, M_{\mathbf{a}}\right)_{0}$ is expressed by an $r \times s$ matrix $\left(h_{i j}\right)$ with $h_{i j} \in R_{a_{i}-b_{j}}$. When $g \in\left(M_{\mathbf{b}}\right)_{0}$ is fixed, the image of the linear map $\operatorname{Hom}\left(M_{\mathbf{b}}, M_{\mathbf{a}}\right)_{0} \rightarrow\left(M_{\mathbf{a}}\right)_{0}$ given by $h \mapsto h(g)$ coincides with $\left(J_{g} M_{\mathbf{a}}\right)_{0}$. The following proposition is then obvious:

Proposition 4.2. The following three conditions on the pair $(f, g)$ of $f \in\left(M_{\mathbf{a}}\right)_{0}^{\text {ci }}$ and $g \in\left(M_{\mathbf{b}}\right)_{0}^{\text {ci }}$ are equivalent:

(i) $X_{\langle f\rangle}$ contains $Z_{\langle g\rangle}$ as a subscheme,

(ii) $f$ is contained in $\left(J_{g} M_{\mathbf{a}}\right)_{0}$,

(iii) there exists an element $h \in \operatorname{Hom}\left(M_{\mathbf{b}}, M_{\mathbf{a}}\right)_{0}$ such that $f=h(g)$.

Let $\mathcal{F}_{\mathbf{b}, \mathbf{a}}$ be the contravariant functor from the category of locally Noetherian schemes over $\mathbb{C}$ to the category of sets that associates to a locally Noetherian scheme $S \rightarrow$ Spec $\mathbb{C}$ the set of pairs $\left(Z_{S}, X_{S}\right)$, where $Z_{S} \subset \mathbb{P}^{n} \times S$ and $X_{S} \subset \mathbb{P}^{n} \times S$ are families of complete intersections in $\mathbb{P}^{n}$ with multi-degrees $\mathbf{b}$ and $\mathbf{a}$, respectively, parametrized by $S$ such that $Z_{S}$ is a subscheme of $X_{S}$. This functor $\mathcal{F}_{\mathbf{b}, \mathbf{a}}$ is represented by a closed subscheme $F_{\mathbf{b}, \mathbf{a}}$ of $H_{n, \mathbf{b}} \times H_{n, \mathbf{a}}$. (The scheme $F_{\mathbf{b}, \mathbf{a}}$ may possibly be empty.) We denote the projections by $\rho^{\prime}: F_{\mathbf{b}, \mathbf{a}} \rightarrow H_{n, \mathbf{b}}$ and $\rho: F_{\mathbf{b}, \mathbf{a}} \rightarrow H_{n, \mathbf{a}}$. The universal family over $F_{\mathbf{b}, \mathbf{a}}$ is the pair $(\widetilde{\mathcal{Z}}, \widetilde{\mathcal{X}})$ of $\widetilde{\mathcal{Z}}:=\mathcal{Z} \times_{H_{n, \mathbf{b}}} F_{\mathbf{b}, \mathbf{a}}$ and $\widetilde{\mathcal{X}}:=\mathcal{X} \times_{H_{n, \mathbf{a}}} F_{\mathbf{b}, \mathbf{a}}$. We denote by $\pi: \widetilde{\mathcal{Z}} \rightarrow F_{\mathbf{b}, \mathbf{a}}$ and $\beta: \widetilde{\mathcal{Z}} \rightarrow \mathcal{Z}$ the natural projections. We also denote by $\alpha: \widetilde{\mathcal{Z}} \rightarrow \mathcal{X}$ the composite of the closed immersion $\widetilde{\mathcal{Z}} \hookrightarrow \widetilde{\mathcal{X}}$ and the natural projection $\widetilde{\mathcal{X}} \rightarrow \mathcal{X}$. Thus we obtain the following commutative diagram:

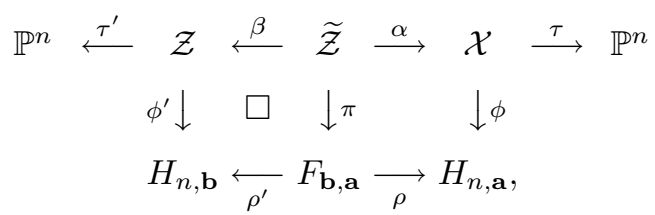

in which $\tau \circ \alpha=\tau^{\prime} \circ \beta$ holds. A point of $\widetilde{\mathcal{Z}}$ is a triple

$$
(p,\langle g\rangle,\langle f\rangle) \in \mathbb{P}^{n} \times H_{n, \mathbf{b}} \times H_{n, \mathbf{a}}
$$

that satisfies $p \in Z_{\langle g\rangle} \subset X_{\langle f\rangle}$. The projection $\pi$ maps $(p,\langle g\rangle,\langle f\rangle)$ to $(\langle g\rangle,\langle f\rangle) \in F_{\mathbf{b}, \mathbf{a}}$, and the morphism $\alpha$ maps $(p,\langle g\rangle,\langle f\rangle)$ to $(p,\langle f\rangle) \in \mathcal{X}$.

The right square of the diagram (4.3) is the universal family of the families (1.2) of complete intersections of multi-degree $\mathbf{b}$ contained in complete intersections of multidegree a. Remark that the linear automorphism group $P G L(n+1)$ of $\mathbb{P}^{n}$ acts on the diagram (4.3). 
REMARK 4.3. If $(n, \mathbf{a}, \mathbf{b})$ satisfies the first inequality $a_{i} \geq b_{i}(i=1, \ldots, r)$ of the condition (2.2) in Main Theorem, then $F_{\mathbf{b}, \mathbf{a}}$ is non-empty. Indeed, we choose linear forms $\ell_{1}, \ldots, \ell_{r}, \ell_{1}^{\prime}, \ldots, \ell_{s}^{\prime} \in R_{1}$ generally. We define $g \in\left(M_{\mathbf{b}}\right)_{0}^{c i}$ by $g_{j}:=\ell_{j}^{\prime b_{j}}$. Since $a_{i} \geq b_{i}$, we can define $f \in\left(M_{\mathbf{a}}\right)_{0}^{c i}$ by $f_{i}:=\ell_{i}^{\prime b_{i}} \ell_{i}^{a_{i}-b_{i}}$. Then $(\langle g\rangle,\langle f\rangle)$ is a point of $F_{\mathbf{b}, \mathbf{a}}$.

From now on to the end of this section, we assume that $F_{\mathbf{b}, \mathbf{a}}$ is non-empty. We define a vector space $U$ with a natural morphism $\nu: U \rightarrow\left(M_{\mathbf{a}}\right)_{0}$ by

$$
U:=\left(M_{\mathbf{b}}\right)_{0} \times \operatorname{Hom}\left(M_{\mathbf{b}}, M_{\mathbf{a}}\right)_{0} \quad \text { and } \quad \nu(g, h):=h(g) .
$$

We then put

$$
U^{c i}:=\left(\left(M_{\mathbf{b}}\right)_{0}^{c i} \times \operatorname{Hom}\left(M_{\mathbf{b}}, M_{\mathbf{a}}\right)_{0}\right) \cap \nu^{-1}\left(\left(M_{\mathbf{a}}\right)_{0}^{c i}\right) .
$$

Note that $U^{c i}$ is a Zariski open subset of $U$, and hence is irreducible. By Proposition 4.2, the map

$$
\sigma(g, h):=(\langle g\rangle,\langle h(g)\rangle)
$$

defines a surjective morphism $\sigma: U^{c i} \rightarrow F_{\mathbf{b}, \mathbf{a}}$, which makes the following diagram commutative:

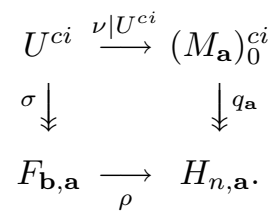

In particular, the scheme $F_{\mathbf{b}, \mathbf{a}}$ is irreducible.

Proposition 4.4. The morphism $\rho^{\prime}: F_{\mathbf{b}, \mathbf{a}} \rightarrow H_{n, \mathbf{b}}$ is smooth.

Proof. For a non-negative integer $k$, we set $A_{k}:=\mathbb{C}[t] /\left(t^{k+1}\right)$, and for a scheme $T$ over $\mathbb{C}$, we denote by $T\left(A_{k}\right)$ the set of $A_{k}$-valued points of $T$. If we are given $\langle g\rangle^{[k+1]} \in$ $H_{n, \mathbf{b}}\left(A_{k+1}\right)$ and $\left(\langle g\rangle^{[k]},\langle f\rangle^{[k]}\right) \in F_{\mathbf{b}, \mathbf{a}}\left(A_{k}\right)$ satisfying $\langle g\rangle^{[k]}=\langle g\rangle^{[k+1]} \bmod t^{k+1}$, it is enough to show that $\left(\langle g\rangle^{[k]},\langle f\rangle^{[k]}\right)$ extends to an element $\left(\langle g\rangle^{[k+1]},\langle f\rangle^{[k+1]}\right)$ of $F_{\mathbf{b}, \mathbf{a}}\left(A_{k+1}\right)$ over the given point $\langle g\rangle^{[k+1]} \in H_{n, \mathbf{b}}\left(A_{k+1}\right)$. Since both of

$$
q_{\mathbf{a}}:\left(M_{\mathbf{a}}\right)_{0}^{c i} \rightarrow H_{n, \mathbf{a}} \quad \text { and } \quad q_{\mathbf{b}}:\left(M_{\mathbf{b}}\right)_{0}^{c i} \rightarrow H_{n, \mathbf{b}}
$$

are smooth, there exist

$$
g^{[k+1]} \in\left(M_{\mathbf{b}}\right)_{0} \otimes_{\mathbb{C}} A_{k+1} \quad \text { and } \quad f^{[k]} \in\left(M_{\mathbf{a}}\right)_{0} \otimes_{\mathbb{C}} A_{k}
$$

that satisfy $q_{\mathbf{b}}\left(g^{[k+1]}\right)=\langle g\rangle^{[k+1]}$ and $q_{\mathbf{a}}\left(f^{[k]}\right)=\langle f\rangle^{[k]}$. We put

$$
g^{[k]}:=g^{[k+1]} \bmod t^{k+1} \in\left(M_{\mathbf{b}}\right)_{0} \otimes_{\mathbb{C}} A_{k},
$$

which satisfies $\left\langle g^{[k]}\right\rangle=\langle g\rangle^{[k]}$. By the definition of $F_{\mathbf{b}, \mathbf{a}}$, the ideal $J_{g^{[k]}}$ of $R \otimes_{\mathbb{C}} A_{k}$ generated by the components of $g^{[k]}$ contains the ideal $J_{f^{[k]}}$. Hence there exists $h^{[k]} \in$ $\operatorname{Hom}\left(M_{\mathbf{b}}, M_{\mathbf{a}}\right)_{0} \otimes_{\mathbb{C}} A_{k}$ such that $f^{[k]}=h^{[k]}\left(g^{[k]}\right)$ holds. Let $h^{[k+1]}$ be any element of $\operatorname{Hom}\left(M_{\mathbf{b}}, M_{\mathbf{a}}\right)_{0} \otimes_{\mathbb{C}} A_{k+1}$ satisfying $h^{[k+1]} \bmod t^{k+1}=h^{[k]}$. We put

$$
f^{[k+1]}:=h^{[k+1]}\left(g^{[k+1]}\right) \in\left(M_{\mathbf{a}}\right)_{0} \otimes_{\mathbb{C}} A_{k+1} .
$$

Since being a complete intersection is an open condition on defining polynomials, the ideal $J_{f[k+1]}$ of $R \otimes_{\mathbb{C}} A_{k+1}$ defines a family of complete intersections of multi-degree a over Spec $A_{k+1}$. Thus $\left(\left\langle g^{[k+1]}\right\rangle,\left\langle f^{[k+1]}\right\rangle\right)$ is the hoped-for $A_{k+1}$-valued point of $F_{\mathbf{b}, \mathbf{a}}$. 


\section{Corollary 4.5.}

(1) The scheme $F_{\mathbf{b}, \mathbf{a}}$ is smooth.

(2) The morphism $\beta: \widetilde{\mathcal{Z}} \rightarrow \mathcal{Z}$ is smooth.

In particular, $\widetilde{\mathcal{Z}}$ is smooth.

Let $(g, h)$ be a point of $U^{c i}$. We have the following natural identifications of vector spaces:

$$
\begin{aligned}
& H^{0}\left(Z_{\langle g\rangle}, \mathcal{N}_{Z_{\langle g\rangle} / \mathbb{P}^{n}}\right)=T_{\langle g\rangle} H_{n, \mathbf{b}}=\left(M_{\mathbf{b}} / J_{g} M_{\mathbf{b}}\right)_{0}, \\
& H^{0}\left(Z_{\langle g\rangle}, \mathcal{N}_{X_{\langle h(g)\rangle} / \mathbb{P}^{n}} \mid Z_{\langle g\rangle}\right)=\left(M_{\mathbf{a}} / J_{g} M_{\mathbf{a}}\right)_{0}, \\
& H^{0}\left(X_{\langle h(g)\rangle}, \mathcal{N}_{X_{\langle h(g)\rangle} / \mathbb{P}^{n}}\right)=T_{\langle h(g)\rangle} H_{n, \mathbf{a}}=\left(M_{\mathbf{a}} / J_{h(g)} M_{\mathbf{a}}\right)_{0} .
\end{aligned}
$$

The restriction homomorphism $\mathcal{N}_{X_{\langle h(g)\rangle} / \mathbb{P}^{n}} \rightarrow \mathcal{N}_{X_{\langle h(g)\rangle} / \mathbb{P}^{n}} \mid Z_{\langle g\rangle}$ of coherent sheaves induces, via (4.6), a linear map

$$
\zeta^{\prime}: T_{\langle h(g)\rangle} H_{n, \mathbf{a}} \rightarrow H^{0}\left(Z_{\langle g\rangle}, \mathcal{N}_{X_{\langle h(g)\rangle} / \mathbb{P}^{n}} \mid Z_{\langle g\rangle}\right) .
$$

Under the identifications (4.6) and (4.5), the linear map $\zeta^{\prime}$ is identified with the natural quotient homomorphism

$$
\left(M_{\mathbf{a}} / J_{h(g)} M_{\mathbf{a}}\right)_{0} \rightarrow\left(M_{\mathbf{a}} / J_{g} M_{\mathbf{a}}\right)_{0} .
$$

In particular, $\zeta^{\prime}$ is surjective. On the other hand, since $Z_{\langle g\rangle}$ is a subscheme of $X_{\langle h(g)\rangle}$, there is a natural homomorphism

$$
\mathcal{N}_{Z_{\langle g\rangle} / \mathbb{P}^{n}} \rightarrow \mathcal{N}_{X_{\langle h(g)\rangle} / \mathbb{P}^{n}} \mid Z_{\langle g\rangle}
$$

of coherent sheaves over $Z_{\langle g\rangle}$, which induces, via (4.4), a linear map

$$
\zeta: T_{\langle g\rangle} H_{n, \mathbf{b}} \rightarrow H^{0}\left(Z_{\langle g\rangle}, \mathcal{N}_{X_{\langle h(g)\rangle} / \mathbb{P}^{n}} \mid Z_{\langle g\rangle}\right) .
$$

Under the identifications (4.4) and (4.5), the linear map $\zeta$ is identified with the homomorphism

$$
\langle h\rangle_{g}:\left(M_{\mathbf{b}} / J_{g} M_{\mathbf{b}}\right)_{0} \rightarrow\left(M_{\mathbf{a}} / J_{g} M_{\mathbf{a}}\right)_{0}
$$

induced from $h: M_{\mathbf{b}} \rightarrow M_{\mathbf{a}}$.

Proposition 4.6. Let $(g, h)$ be a point of $U^{c i}$, and $P$ the point $\sigma(g, h)=(\langle g\rangle,\langle h(g)\rangle)$ of $F_{\mathbf{b}, \mathbf{a}}$. Then we have the following diagram of fiber product:

$$
\begin{array}{ccc}
T_{P} F_{\mathbf{b}, \mathbf{a}} & \stackrel{(d \rho)_{P}}{\longrightarrow} & T_{\langle h(g)\rangle} H_{n, \mathbf{a}} \\
\left(d \rho^{\prime}\right)_{P} \downarrow & \square & \downarrow^{\zeta^{\prime}} \\
T_{\langle g\rangle} H_{n, \mathbf{b}} & \underset{\zeta}{\longrightarrow} H^{0}\left(Z_{\langle g\rangle}, \mathcal{N}_{X\langle h(g)\rangle} / \mathbb{P}^{n} \mid Z_{\langle g\rangle}\right) .
\end{array}
$$

Proof. By the identifications (4.4) and (4.6), any vectors of $T_{\langle g\rangle} H_{n, \mathbf{b}}$ and $T_{\langle h(g)\rangle} H_{n, \mathbf{a}}$ are given as elements

$$
\bar{g}^{\prime}:=g^{\prime} \bmod \left(J_{g} M_{\mathbf{b}}\right)_{0} \quad \text { and } \quad \bar{f}^{\prime}:=f^{\prime} \bmod \left(J_{h(g)} M_{\mathbf{a}}\right)_{0}
$$

of $\left(M_{\mathbf{b}} / J_{g} M_{\mathbf{b}}\right)_{0}$ and $\left(M_{\mathbf{a}} / J_{h(g)} M_{\mathbf{a}}\right)_{0}$ by some $g^{\prime} \in\left(M_{\mathbf{b}}\right)_{0}$ and $f^{\prime} \in\left(M_{\mathbf{a}}\right)_{0}$, respectively. Let $\varepsilon$ be a dual number: $\varepsilon^{2}=0$. The vectors $\bar{g}^{\prime}$ and $\bar{f}^{\prime}$ correspond to the infinitesimal 
displacements

$$
Z_{\left\langle g+\varepsilon g^{\prime}\right\rangle} \rightarrow \operatorname{Spec} \mathbb{C}[\varepsilon] \quad \text { and } \quad X_{\left\langle h(g)+\varepsilon f^{\prime}\right\rangle} \rightarrow \operatorname{Spec} \mathbb{C}[\varepsilon]
$$

of $Z_{\langle g\rangle}$ and $X_{\langle h(g)\rangle}$ defined by the homogeneous ideals $J_{g}+\varepsilon J_{g^{\prime}}$ and $J_{h(g)}+\varepsilon J_{f^{\prime}}$ of $R \otimes_{\mathbb{C}} \mathbb{C}[\varepsilon]$, respectively. Then the vector $\left(\bar{g}^{\prime}, \bar{f}^{\prime}\right)$, regarded as a tangent vector to $H_{n, \mathbf{b}} \times H_{n, \mathbf{a}}$ at $(\langle g\rangle,\langle h(g)\rangle)$, is tangent to $F_{\mathbf{b}, \mathbf{a}}$ if and only if $Z_{\left\langle g+\varepsilon g^{\prime}\right\rangle}$ is contained in $X_{\left\langle h(g)+\varepsilon f^{\prime}\right\rangle}$ as a subscheme; that is, there exist elements $h_{1}, h_{2} \in \operatorname{Hom}\left(M_{\mathbf{b}}, M_{\mathbf{a}}\right)_{0}$ such that $h_{1}+\varepsilon h_{2} \in \operatorname{Hom}\left(M_{\mathbf{b}}, M_{\mathbf{a}}\right)_{0} \otimes_{\mathbb{C}} \mathbb{C}[\varepsilon]$ satisfies

$$
\left(h_{1}+\varepsilon h_{2}\right)\left(g+\varepsilon g^{\prime}\right)=h(g)+\varepsilon f^{\prime} .
$$

Suppose that $h_{1}+\varepsilon h_{2}$ satisfies (4.8). Because $h_{1}(g)=h(g)$, each row vector of the matrix $h_{1}-h$ is contained in the syzygy of the regular sequence $\left(g_{1}, \ldots, g_{s}\right)$, and hence every component of $h-h_{1}$ is contained in $J_{g}$. Therefore the two linear maps $\langle h\rangle_{g}$ and $\left\langle h_{1}\right\rangle_{g}$ from $\left(M_{\mathbf{b}} / J_{g} M_{\mathbf{b}}\right)_{0}$ to $\left(M_{\mathbf{a}} / J_{g} M_{\mathbf{a}}\right)_{0}$ are the same. The equality $h_{1}\left(g^{\prime}\right)+h_{2}(g)=f^{\prime}$ then tells us that $f^{\prime} \bmod \left(J_{g} M_{\mathbf{a}}\right)_{0}$ is equal to $\langle h\rangle_{g}\left(\bar{g}^{\prime}\right)$, because $h_{2}(g) \in\left(J_{g} M_{\mathbf{a}}\right)_{0}$. Hence $\left(\bar{g}^{\prime}, \bar{f}^{\prime}\right)$ is contained in the fiber product of $\zeta$ and $\zeta^{\prime}$. Conversely, if $\left(\bar{g}^{\prime}, \bar{f}^{\prime}\right)$ is contained in the fiber product of $\zeta$ and $\zeta^{\prime}$, then it is easy to find an element $h_{2} \in \operatorname{Hom}\left(M_{\mathbf{b}}, M_{\mathbf{a}}\right)_{0}$ satisfying $\left(h+\varepsilon h_{2}\right)\left(g+\varepsilon g^{\prime}\right)=h(g)+\varepsilon f^{\prime}$.

Since $F_{\mathbf{b}, \mathbf{a}}$ is reduced by Corollary $4.5(1)$, we obtain the following:

Corollary 4.7. Let $(g, h)$ be an arbitrary point of $U^{c i}$.

(1) The dimension of $F_{\mathbf{b}, \mathbf{a}}$ is equal to

$$
\begin{aligned}
\operatorname{dim}\left(M_{\mathbf{a}} / J_{h(g)} M_{\mathbf{a}}\right)_{0}+\operatorname{dim}\left(M_{\mathbf{b}} / J_{g} M_{\mathbf{b}}\right)_{0}-\operatorname{dim}\left(M_{\mathbf{a}} / J_{g} M_{\mathbf{a}}\right)_{0} \\
=\operatorname{dim} H_{n, \mathbf{a}}+\operatorname{dim} H_{n, \mathbf{b}}-\operatorname{dim}\left(M_{\mathbf{a}} / J_{g} M_{\mathbf{a}}\right)_{0} .
\end{aligned}
$$

(2) Let $P$ be the point $\sigma(g, h)$ of $F_{\mathbf{b}, \mathbf{a}}$. Then the dimension of the cokernel of the linear $\operatorname{map}(d \rho)_{P}: T_{P} F_{\mathbf{b}, \mathbf{a}} \rightarrow T_{\langle h(g)\rangle} H_{n, \mathbf{a}}$ is equal to

$$
\operatorname{dim} \operatorname{Coker} \zeta=\operatorname{dim} \operatorname{Coker}\langle h\rangle_{g}=\operatorname{dim}\left(M_{\mathbf{a}} /\left(J_{g} M_{\mathbf{a}}+h\left(M_{\mathbf{b}}\right)\right)\right)_{0} .
$$

Proposition 4.8. Let $(g, h)$ be a point of $U^{c i}$, and let $p$ be a point of $Z_{\langle g\rangle}$. We put

$$
Q:=(p,\langle g\rangle,\langle h(g)\rangle),
$$

which is a point of $\widetilde{\mathcal{Z}}$. Let $I_{p}$ denote the homogeneous ideal of $R$ defining the point $p$. Then the dimension of the kernel of $(d \alpha)_{Q}: T_{Q} \widetilde{\mathcal{Z}} \rightarrow T_{\alpha(Q)} \mathcal{X}$ is equal to

$$
\operatorname{dim} F_{\mathbf{b}, \mathbf{a}}-\operatorname{dim} H_{n, \mathbf{a}}-s+\operatorname{dim}\left(M_{\mathbf{a}} /\left(J_{g} M_{\mathbf{a}}+I_{p} h\left(M_{\mathbf{b}}\right)\right)\right)_{0} .
$$

Proof. Since $\widetilde{\mathcal{Z}}$ is a closed subscheme of $H_{n, \mathbf{b}} \times \mathcal{X}$ with $\rho^{\prime} \circ \pi$ and $\alpha$ being the projections, the kernel of $(d \alpha)_{Q}$ is mapped isomorphically to a subspace of $T_{\langle g\rangle} H_{n, \mathbf{b}}$ by the linear map $d\left(\rho^{\prime} \circ \pi\right)_{Q}$. We will show that this subspace

$$
\left(d\left(\rho^{\prime} \circ \pi\right)_{Q}\right)\left(\operatorname{Ker}(d \alpha)_{Q}\right) \subset T_{\langle g\rangle} H_{n, \mathbf{b}}
$$

coincides with the subspace

$$
\left(I_{p} M_{\mathbf{b}} / J_{g} M_{\mathbf{b}}\right)_{0} \cap \operatorname{Ker}\langle h\rangle_{g} \subset\left(M_{\mathbf{b}} / J_{g} M_{\mathbf{b}}\right)_{0}
$$

under the identification (4.4). Let $g^{\prime}$ be an element of $\left(M_{\mathbf{b}}\right)_{0}$. We put

$$
\bar{g}^{\prime}:=g^{\prime} \bmod \left(J_{g} M_{\mathbf{b}}\right)_{0} \in\left(M_{\mathbf{b}} / J_{g} M_{\mathbf{b}}\right)_{0},
$$


which gives the corresponding displacement $Z_{\left\langle g+\varepsilon g^{\prime}\right\rangle} \rightarrow \operatorname{Spec} \mathbb{C}[\varepsilon]$ of $Z_{\langle g\rangle}$. The subspace (4.11) consists of vectors corresponding to infinitesimal displacements with $p$ in $Z_{\left\langle g+\varepsilon g^{\prime}\right\rangle}$ and with $Z_{\left\langle g+\varepsilon g^{\prime}\right\rangle}$ remaining in $X_{\langle h(g)\rangle}$. The displacement $Z_{\left\langle g+\varepsilon g^{\prime}\right\rangle}$ contains $p$ if and only if $J_{g^{\prime}} \subset I_{p}$ holds, which is equivalent to $\bar{g}^{\prime} \in\left(I_{p} M_{\mathbf{b}} / J_{g} M_{\mathbf{b}}\right)_{0}$. On the other hand, by Proposition 4.6, the displacement $Z_{\left\langle g+\varepsilon g^{\prime}\right\rangle}$ remains in $X_{\langle h(g)\rangle}$ if and only if the corresponding vector of $T_{\langle g\rangle} H_{n, \mathbf{b}}$ is contained in $\operatorname{Ker} \zeta$. Since $\zeta$ is identified with $\langle h\rangle_{g}$, this holds if and only if $\bar{g}^{\prime} \in \operatorname{Ker}\langle h\rangle_{g}$. Therefore (4.11) coincides with (4.12) by (4.4). The cokernel of the homomorphism

$$
\left(I_{p} M_{\mathbf{b}} / J_{g} M_{\mathbf{b}}\right)_{0} \hookrightarrow\left(M_{\mathbf{b}} / J_{g} M_{\mathbf{b}}\right)_{0} \stackrel{\langle h\rangle_{g}}{\longrightarrow}\left(M_{\mathbf{a}} / J_{g} M_{\mathbf{a}}\right)_{0}
$$

is $\left(M_{\mathbf{a}} /\left(J_{g} M_{\mathbf{a}}+I_{p} h\left(M_{\mathbf{b}}\right)\right)\right)_{0}$. On the other hand, $\operatorname{dim}\left(M_{\mathbf{b}} / I_{p} M_{\mathbf{b}}\right)_{0}$ is equal to $s$. These lead us to the conclusion that $\operatorname{dim} \operatorname{Ker}(d \alpha)_{Q}$ is equal to

$$
\operatorname{dim}\left(M_{\mathbf{b}} / J_{g} M_{\mathbf{b}}\right)_{0}-s-\operatorname{dim}\left(M_{\mathbf{a}} / J_{g} M_{\mathbf{a}}\right)_{0}+\operatorname{dim}\left(M_{\mathbf{a}} /\left(J_{g} M_{\mathbf{a}}+I_{p} h\left(M_{\mathbf{b}}\right)\right)\right)_{0},
$$

which coincides with (4.10) by Corollary $4.7(1)$.

In the sequel, we use the following notation. For positive integers $d$ and $e$, let $\operatorname{Mat}(d, e)$ denote the vector space of all $d \times e$ matrices with entries in $\mathbb{C}$, and $D(d, e)$ the Zariski closed subset of $\operatorname{Mat}(d, e)$ consisting of matrices whose rank is less than $\min (d, e)$. It is easy to see that $D(d, e)$ is irreducible. We set

$$
o:=[1: 0: \ldots: 0] \in \mathbb{P}^{n} .
$$

For a homogeneous polynomial $a \in R$, we put

$$
a(o):=\text { the coefficient of } x_{0}^{\operatorname{deg} a} \text { in } a .
$$

Let $I_{o}$ be the homogeneous ideal of $R$ defining $o$ in $\mathbb{P}^{n}$ :

$$
I_{o}:=\left\langle x_{1}, \ldots, x_{n}\right\rangle \subset R .
$$

We define linear maps $\lambda_{i}:\left(I_{o} M_{\mathbf{a}}\right)_{0} \rightarrow \mathbb{C}^{n}$ for $i=1, \ldots, r$ and $\mu_{j}:\left(I_{o} M_{\mathbf{b}}\right)_{0} \rightarrow \mathbb{C}^{n}$ for $j=1, \ldots, s$ by

$$
\lambda_{i}(f):=\left(\frac{\partial f_{i}}{\partial x_{1}}(o), \ldots, \frac{\partial f_{i}}{\partial x_{n}}(o)\right) \text { and } \quad \mu_{j}(g):=\left(\frac{\partial g_{j}}{\partial x_{1}}(o), \ldots, \frac{\partial g_{j}}{\partial x_{n}}(o)\right) .
$$

Let $\lambda:\left(I_{o} M_{\mathbf{a}}\right)_{0} \rightarrow \operatorname{Mat}(r, n)$ and $\mu:\left(I_{o} M_{\mathbf{b}}\right)_{0} \rightarrow \operatorname{Mat}(s, n)$ be linear maps defined by

$$
\lambda(f):=\left(\begin{array}{c}
\lambda_{1}(f) \\
\vdots \\
\lambda_{r}(f)
\end{array}\right) \quad \text { and } \quad \mu(g):=\left(\begin{array}{c}
\mu_{1}(g) \\
\vdots \\
\mu_{s}(g)
\end{array}\right)
$$

Both of $\lambda$ and $\mu$ are surjective. We define a linear map $\eta: \operatorname{Hom}\left(M_{\mathbf{b}}, M_{\mathbf{a}}\right)_{0} \rightarrow \operatorname{Mat}(r, s)$ by

$$
\eta(h):=\left(h_{i j}(o)\right)
$$

when $h$ is expressed by an $r \times s$ matrix $\left(h_{i j}\right)$ with $h_{i j} \in R_{a_{i}-b_{j}}$. Note that, if $g$ is an element of $\left(I_{o} M_{\mathbf{b}}\right)_{0}$, then, for any $h \in \operatorname{Hom}\left(M_{\mathbf{b}}, M_{\mathbf{a}}\right)_{0}$, we have $h(g) \in\left(I_{o} M_{\mathbf{a}}\right)_{0}$ and $\lambda(h(g))=\eta(h) \cdot \mu(g)$. 
We define an $R$-submodule $N_{\mathbf{a}}$ of $M_{\mathbf{a}}$ by

$$
N_{\mathbf{a}}:=\bigoplus_{i=1}^{r-1} R\left(a_{i}\right) \oplus I_{o}\left(a_{r}\right) .
$$

Note that $\operatorname{Ker} \lambda_{r}=\left(I_{o} N_{\mathbf{a}}\right)_{0}$ holds in $\left(I_{o} M_{\mathbf{a}}\right)_{0}$, and that an element $h$ of $\operatorname{Hom}\left(M_{\mathbf{b}}, M_{\mathbf{a}}\right)_{0}$ is contained in $\operatorname{Hom}\left(M_{\mathbf{b}}, N_{\mathbf{a}}\right)_{0}$ if and only if the $r$-th row vector of $\eta(h)$ is the zero vector. We put

$$
\begin{aligned}
& \left(I_{o} M_{\mathbf{b}}\right)_{0}^{c i}:=\left(I_{o} M_{\mathbf{b}}\right)_{0} \cap\left(M_{\mathbf{b}}\right)_{0}^{c i}, \\
& \left(I_{o} M_{\mathbf{a}}\right)_{0}^{c i}:=\left(I_{o} M_{\mathbf{a}}\right)_{0} \cap\left(M_{\mathbf{a}}\right)_{0}^{c i}, \\
& \left(I_{o} N_{\mathbf{a}}\right)_{0}^{c i}:=\left(I_{o} N_{\mathbf{a}}\right)_{0} \cap\left(M_{\mathbf{a}}\right)_{0}^{c i} .
\end{aligned}
$$

For $f \in\left(M_{\mathbf{a}}\right)_{0}^{c i}$ and $g \in\left(M_{\mathbf{b}}\right)_{0}^{c i}$, we have the following:

$$
(o,\langle f\rangle) \in \mathcal{X} \Longleftrightarrow f \in\left(I_{o} M_{\mathbf{a}}\right)_{0}^{c i}, \quad(o,\langle g\rangle) \in \mathcal{Z} \Longleftrightarrow g \in\left(I_{o} M_{\mathbf{b}}\right)_{0}^{c i} .
$$

Let $\Gamma$ be the Zariski closed subset of $\mathcal{X}$ consisting of critical points of $\phi: \mathcal{X} \rightarrow H_{n, \mathbf{a}}$, and $\Gamma^{\prime}$ the Zariski closed subset of $\mathcal{Z}$ consisting of critical points of $\phi^{\prime}: \mathcal{Z} \rightarrow H_{n, \mathbf{b}}$. We put

$$
\Gamma_{o}:=\tau^{-1}(o) \cap \Gamma, \quad \Gamma_{o}^{\prime}:=\tau^{\prime-1}(o) \cap \Gamma^{\prime} .
$$

For $f \in\left(I_{o} M_{\mathbf{a}}\right)_{0}^{c i}$ and $g \in\left(I_{o} M_{\mathbf{b}}\right)_{0}^{c i}$, we have the following:

$$
\begin{gathered}
(o,\langle f\rangle) \in \Gamma_{o} \Longleftrightarrow \lambda(f) \in D(r, n), \\
(o,\langle g\rangle) \in \Gamma_{o}^{\prime} \Longleftrightarrow \mu(g) \in D(s, n) .
\end{gathered}
$$

If $f \in\left(I_{o} N_{\mathbf{a}}\right)_{0}^{c i}$, then $\lambda(f) \in D(r, n)$. Hence we can define a morphism $\gamma:\left(I_{o} N_{\mathbf{a}}\right)_{0}^{c i} \rightarrow \Gamma_{o}$ by

$$
\gamma(f):=(o,\langle f\rangle)
$$

Proposition 4.9. The Zariski closed subset $\Gamma_{o}$ of $\mathcal{X}$ is irreducible, and the morphism $\gamma:\left(I_{o} N_{\mathbf{a}}\right)_{0}^{c i} \rightarrow \Gamma_{o}$ is dominant.

Proof. By (4.13) and (4.14), the map $f \mapsto(o,\langle f\rangle)$ gives a surjective morphism from $\lambda^{-1}(D(r, n)) \cap\left(I_{o} M_{\mathbf{a}}\right)_{0}^{c i}$ to $\Gamma_{o}$. Because $\lambda$ is a surjective linear map and $D(r, n)$ is irreducible, $\lambda^{-1}(D(r, n))$ is also irreducible. Since $\lambda^{-1}(D(r, n)) \cap\left(I_{o} M_{\mathbf{a}}\right)_{0}^{c i}$ is Zariski open in $\lambda^{-1}(D(r, n)), \Gamma_{o}$ is also irreducible. Let $f$ be a general element of $\lambda^{-1}(D(r, n))$. Then $\lambda(f)$ is of rank $r-1$, and the vector $\lambda_{r}(f)$ can be written as a linear combination of $\lambda_{1}(f), \ldots, \lambda_{r-1}(f)$. Since $a_{r} \geq a_{i}$ for $i<r$, there exist homogeneous polynomials $c_{1}, \ldots, c_{r-1}$ with $c_{i} \in R_{a_{r}-a_{i}}$ such that, if we put

$$
f_{r}^{\prime}:=f_{r}-c_{1} f_{1}-\ldots-c_{r-1} f_{r-1} \quad \text { and } \quad f^{\prime}:=\left(f_{1}, \ldots, f_{r-1}, f_{r}^{\prime}\right)^{T}
$$

then $\lambda_{r}\left(f^{\prime}\right)=0$ holds, which means $f^{\prime} \in\left(I_{o} N_{\mathbf{a}}\right)_{0}$. From $J_{f}=J_{f^{\prime}}$, we conclude that $(o,\langle f\rangle)=\left(o,\left\langle f^{\prime}\right\rangle\right)$ belongs to the image of $\gamma$. Since $(o,\langle f\rangle)$ is a general point of $\Gamma_{o}$, the morphism $\gamma$ is dominant.

REMARK 4.10. The irreducibility of $\Gamma$ and that of $S_{n, \mathbf{a}}=\phi(\Gamma)$ follow from Proposition 4.9 and the action of $P G L(n+1)$ on the diagram (4.3). 
Corollary 4.11. Suppose that $(o,\langle f\rangle)$ is a general point of $\Gamma_{o}$. Then the singular locus of $X_{\langle f\rangle}$ consists of only one point o, which is a hypersurface singularity of $X_{\langle f\rangle}$ with non-degenerate Hessian.

We put

$$
\Xi:=\alpha^{-1}(\Gamma) \backslash\left(\alpha^{-1}(\Gamma) \cap \beta^{-1}\left(\Gamma^{\prime}\right)\right) \text { and } \Xi_{o}:=(\tau \circ \alpha)^{-1}(o) \cap \Xi,
$$

which are locally closed subsets of $\widetilde{\mathcal{Z}}$ (possibly empty). A point $(o,\langle g\rangle,\langle f\rangle)$ of the locus $(\tau \circ \alpha)^{-1}(o) \subset \widetilde{\mathcal{Z}}$ is contained in $\Xi_{o}$ if and only if $X_{\langle f\rangle}$ is singular at $o$ and $Z_{\langle g\rangle}$ is smooth at $o$. The morphism $\alpha: \widetilde{\mathcal{Z}} \rightarrow \mathcal{X}$ induces a morphism $\alpha \mid \Xi_{o}: \Xi_{o} \rightarrow \Gamma_{o}$.

REMARK 4.12. Invoking the action of $P G L(n+1)$ on the diagram (4.3), we can paraphrase the second condition of Main Theorem into the condition that $\alpha \mid \Xi_{o}: \Xi_{o} \rightarrow \Gamma_{o}$ is dominant.

We define a linear subspace $V$ of $U=\left(M_{\mathbf{b}}\right)_{0} \times \operatorname{Hom}\left(M_{\mathbf{b}}, M_{\mathbf{a}}\right)_{0}$ by

$$
V:=\left(I_{o} M_{\mathbf{b}}\right)_{0} \times \operatorname{Hom}\left(M_{\mathbf{b}}, N_{\mathbf{a}}\right)_{0} .
$$

We then put $V^{c i}:=V \cap U^{c i}$ and

$$
V^{\natural}:=\left\{(g, h) \in V^{c i} \mid \mu(g) \notin D(s, n)\right\}=\left\{(g, h) \in V^{c i} \mid Z_{\langle g\rangle} \text { is smooth at } o\right\} .
$$

By definition, $V^{\natural}$ is Zariski open in the vector space $V$, but may possibly be empty. Recall that $\nu: U \rightarrow\left(M_{\mathbf{a}}\right)_{0}$ is the morphism defined by $\nu(g, h)=h(g)$. We have a morphism

$$
\nu \mid V: V \rightarrow\left(I_{o} N_{\mathbf{a}}\right)_{0} \quad \text { and } \quad \nu \mid V^{\natural}: V^{\natural} \rightarrow\left(I_{o} N_{\mathbf{a}}\right)_{0}^{c i},
$$

which are the restrictions of $\nu$ to $V$ and $V^{\natural}$, respectively. By definition again, if $(g, h) \in V^{\natural}$, then $(o,\langle g\rangle,\langle h(g)\rangle) \in \Xi_{o}$. Let $\xi: V^{\natural} \rightarrow \Xi_{o}$ be the morphism defined by

$$
\xi(g, h):=(o,\langle g\rangle,\langle h(g)\rangle) .
$$

Then we obtain the following commutative diagram:

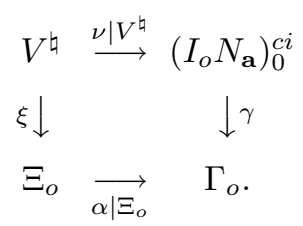

Proposition 4.13. The morphism $\alpha \mid \Xi_{o}: \Xi_{o} \rightarrow \Gamma_{o}$ is dominant if and only if the morphism $\nu \mid V^{\natural}: V^{\natural} \rightarrow\left(I_{o} N_{\mathbf{a}}\right)_{0}^{c i}$ is dominant.

Proof. Since $\gamma$ is dominant by Proposition 4.9, the commutativity of the diagram (4.15) implies that, if $\nu \mid V^{\natural}$ is dominant, then so is $\alpha \mid \Xi_{o}$. Suppose conversely that $\alpha \mid \Xi_{o}$ is dominant. Let $f$ be a general point of $\left(I_{o} N_{\mathbf{a}}\right)_{0}^{c i}$. Since $\gamma$ is dominant, $(o,\langle f\rangle)$ is a general point of $\Gamma_{o}$, and hence $(o,\langle f\rangle)$ is in the image of $\alpha \mid \Xi_{o}$. Thus there exists an element $g \in\left(I_{o} M_{\mathbf{b}}\right)_{0}^{c i}$ such that $(o,\langle g\rangle,\langle f\rangle) \in \Xi_{o}$, which implies that $\mu(g)$ is not contained in $D(s, n)$, and that there exists an element $h \in \operatorname{Hom}\left(M_{\mathbf{b}}, M_{\mathbf{a}}\right)_{0}$ that satisfies $h(g)=f$. From $\lambda_{r}(f)=0$ and $\eta(h) \cdot \mu(g)=\lambda(f)$, the linear independence of the row vectors of $\mu(g)$ implies that the $r$-th row vector of $\eta(h)$ is a zero vector. Therefore $h$ is in fact an element of $\operatorname{Hom}\left(M_{\mathbf{b}}, N_{\mathbf{a}}\right)_{0}$, which means $(g, h) \in V^{\natural}$. Hence the general point $f=h(g)$ of $\left(I_{o} N_{\mathbf{a}}\right)_{0}^{c i}$ is contained in the image of $\nu \mid V^{\natural}$. 
Proposition 4.14. Suppose that $\alpha \mid \Xi_{o}: \Xi_{o} \rightarrow \Gamma_{o}$ is dominant. Then there exists a unique irreducible component $\Xi_{o}^{\prime}$ of $\Xi_{o}$ such that the restriction $\alpha \mid \Xi_{o}^{\prime}: \Xi_{o}^{\prime} \rightarrow \Gamma_{o}$ of $\alpha \mid \Xi_{o}$ to $\Xi_{o}^{\prime}$ is dominant. The closure of the image of $\xi: V^{\natural} \rightarrow \Xi_{o}$ in $\Xi_{o}$ coincides with $\Xi_{o}^{\prime}$.

Proof. Since $\Gamma_{o}$ is irreducible, there exists at least one irreducible component $\Xi_{o}^{\prime}$ of $\Xi_{o}$ that is mapped dominantly onto $\Gamma_{o}$ by $\alpha \mid \Xi_{o}$. Let $(o,\langle g\rangle,\langle f\rangle)$ be a general point of $\Xi_{o}^{\prime}$. Then $\alpha(o,\langle g\rangle,\langle f\rangle)=(o,\langle f\rangle)$ is a general point of $\Gamma_{o}$. Since $\gamma$ is dominant, we can assume that $(o,\langle f\rangle)$ is in the image of $\gamma$; that is, $f$ is an element of $\left(I_{o} N_{\mathbf{a}}\right)_{0}^{c i}$. Let $h \in \operatorname{Hom}\left(M_{\mathbf{b}}, M_{\mathbf{a}}\right)_{0}$ be a homomorphism satisfying $h(g)=f$. From $\mu(g) \notin D(s, n)$ and $\lambda_{r}(f)=0$, we see that $h$ actually is an element of $\operatorname{Hom}\left(M_{\mathbf{b}}, N_{\mathbf{a}}\right)_{0}$. Hence $(g, h)$ is a point of $V^{\natural}$, which is mapped to the general point $(o,\langle g\rangle,\langle f\rangle)$ of $\Xi_{o}^{\prime}$ by $\xi$. Therefore $\Xi_{o}^{\prime}$ is the closure of the image of $\xi: V^{\natural} \rightarrow \Xi_{o}$ in $\Xi_{o}$. Since $V^{\natural}$ is irreducible, the uniqueness of $\Xi_{o}^{\prime}$, as well as the second assertion, is proved.

For an element $(g, h)$ of $U$, we define a linear map $\delta_{(g, h)}: U \rightarrow\left(M_{\mathbf{a}}\right)_{0}$ by

$$
\delta_{(g, h)}(G, H):=H(g)+h(G) \quad\left(G \in\left(M_{\mathbf{b}}\right)_{0}, H \in \operatorname{Hom}\left(M_{\mathbf{b}}, M_{\mathbf{a}}\right)_{0}\right) .
$$

Under the natural isomorphisms $T_{(g, h)} U \cong U$ and $T_{\nu(g, h)}\left(M_{\mathbf{a}}\right)_{0} \cong\left(M_{\mathbf{a}}\right)_{0}$, the linear map $\delta_{(g, h)}$ is equal to

By definition, we have

$$
(d \nu)_{(g, h)}: T_{(g, h)} U \rightarrow T_{\nu(g, h)}\left(M_{\mathbf{a}}\right)_{0}
$$

$$
\begin{aligned}
\delta_{(g, h)}(U) & =\left(J_{g} M_{\mathbf{a}}+h\left(M_{\mathbf{b}}\right)\right)_{0}, \\
\delta_{(g, h)}(V) & =\left(J_{g} N_{\mathbf{a}}+I_{o} h\left(M_{\mathbf{b}}\right)\right)_{0}, \quad \text { and } \\
(g, h) \in V & \Longrightarrow \delta_{(g, h)}(U) \subseteq\left(N_{\mathbf{a}}\right)_{0}, \delta_{(g, h)}(V) \subseteq\left(I_{o} N_{\mathbf{a}}\right)_{0} .
\end{aligned}
$$

Proposition 4.15. Suppose $a_{r} \geq b_{s}$. Then the following conditions on $(n, \mathbf{a}, \mathbf{b})$ are equivalent to each other:

(i) The morphism $\alpha \mid \Xi_{o}: \Xi_{o} \rightarrow \Gamma_{o}$ is dominant.

(ii) If $(g, h) \in V$ is general, then $\delta_{(g, h)}(V)$ coincides with $\left(I_{o} N_{\mathbf{a}}\right)_{0}$.

(iii) If $(g, h) \in V$ is general, then

$$
\operatorname{dim}\left(M_{\mathbf{a}} /\left(J_{g} M_{\mathbf{a}}+I_{o} h\left(M_{\mathbf{b}}\right)\right)\right)_{0}=n+r-s .
$$

(iv) There exists at least one $(g, h) \in V$ such that

$$
\operatorname{dim}\left(M_{\mathbf{a}} /\left(J_{g} M_{\mathbf{a}}+I_{o} h\left(M_{\mathbf{b}}\right)\right)\right)_{0} \leq n+r-s .
$$

Proof. First we show the following:

Claim.

(1) For any $(g, h) \in V, \operatorname{dim}\left(M_{\mathbf{a}} /\left(J_{g} M_{\mathbf{a}}+I_{o} h\left(M_{\mathbf{b}}\right)\right)\right)_{0}$ is larger than or equal to $n+r-s$.

(2) If $(g, h) \in V$ is chosen generally, then $\operatorname{dim}\left(J_{g} M_{\mathbf{a}}+I_{o} h\left(M_{\mathbf{b}}\right)\right)_{0}$ is equal to $\operatorname{dim}\left(J_{g} N_{\mathbf{a}}+I_{o} h\left(M_{\mathbf{b}}\right)\right)_{0}+s$.

Let $(g, h)$ be an arbitrary element of $V$. Then $\left(I_{o} h\left(M_{\mathbf{b}}\right)\right)_{0}$ is contained in the space $\left(I_{o} N_{\mathbf{a}}\right)_{0}=\operatorname{Ker} \lambda_{r}$. On the other hand, if $f \in\left(J_{g} M_{\mathbf{a}}\right)_{0}$, then the $r$-th component $f_{r}$ of $f$ is written as $g_{1} k_{1}+\ldots+g_{s} k_{s}$ with $k_{j} \in R_{a_{r}-b_{j}}$, and $\lambda_{r}(f)$ is equal to

$$
k_{1}(o) \mu_{1}(g)+\ldots+k_{s}(o) \mu_{s}(g) .
$$


Hence the image of $\left(J_{g} M_{\mathbf{a}}+I_{o} h\left(M_{\mathbf{b}}\right)\right)_{0}$ by $\lambda_{r}$ is spanned by $\mu_{1}(g), \ldots, \mu_{s}(g)$, and therefore is of dimension not greater than $s$. On the other hand, $\operatorname{Ker} \lambda_{r}=\left(I_{o} N_{\mathbf{a}}\right)_{0}$ is of codimension $n+r$ in $\left(M_{\mathbf{a}}\right)_{0}$. Hence we obtain

$$
\operatorname{dim}\left(J_{g} M_{\mathbf{a}}+I_{o} h\left(M_{\mathbf{b}}\right)\right)_{0} \leq \operatorname{dim} \operatorname{Ker} \lambda_{r}+s=\operatorname{dim}\left(M_{\mathbf{a}}\right)_{0}-n-r+s,
$$

which implies Claim (1).

Let $(g, h)$ be a general element of $V$. Because $g$ is general in $\left(I_{o} M_{\mathbf{b}}\right)_{0}$, the vectors $\mu_{1}(g), \ldots, \mu_{s}(g)$ are linearly independent. Let $f$ be an element of $\left(J_{g} M_{\mathbf{a}}\right)_{0}$. By the assumption $a_{r} \geq b_{s}$, the degrees $a_{r}-b_{j}$ of the polynomials $k_{j}$ in the expression $f_{r}=g_{1} k_{1}+\ldots+g_{s} k_{s}$ are non-negative for all $j \leq s$. Therefore the coefficients $k_{j}(o)$ in (4.19) can take any values. Hence the image of $\left(J_{g} M_{\mathbf{a}}+I_{o} h\left(M_{\mathbf{b}}\right)\right)_{0}$ by $\lambda_{r}$ is of dimension exactly $s$. Moreover, if $f \in \operatorname{Ker} \lambda_{r}$, then $k_{1}(o)=\ldots=k_{s}(o)=0$. Hence Ker $\lambda_{r} \subseteq\left(J_{g} N_{\mathbf{a}}\right)_{0}$. Because $\left(I_{o} h\left(M_{\mathbf{b}}\right)\right)_{0} \subseteq \operatorname{Ker} \lambda_{r}$, we have

$$
\left(J_{g} M_{\mathbf{a}}+I_{o} h\left(M_{\mathbf{b}}\right)\right)_{0} \cap \operatorname{Ker} \lambda_{r}=\left(J_{g} N_{\mathbf{a}}+I_{o} h\left(M_{\mathbf{b}}\right)\right)_{0} .
$$

Therefore Claim (2) is proved.

Since $\operatorname{dim}\left(M_{\mathbf{a}} /\left(J_{g} M_{\mathbf{a}}+I_{o} h\left(M_{\mathbf{b}}\right)\right)\right)_{0}$ is an upper semi-continuous function of $(g, h)$, Claim (1) implies that conditions (iii) and (iv) are equivalent. By (4.17) and (4.18), the following inequality holds for any $(g, h) \in V$ :

$$
\begin{aligned}
\operatorname{dim}\left(M_{\mathbf{a}}\right)_{0} / \delta_{(g, h)}(V)=\operatorname{dim}\left(M_{\mathbf{a}} /\left(J_{g} N_{\mathbf{a}}+I_{o} h\left(M_{\mathbf{b}}\right)\right)\right)_{0} & \\
& \geq \operatorname{dim}\left(M_{\mathbf{a}} / I_{o} N_{\mathbf{a}}\right)_{0}=n+r .
\end{aligned}
$$

Condition (ii) is satisfied if and only if the equality in (4.20) holds for a general $(g, h) \in V$. The equivalence of conditions (ii) and (iii) now follows from Claim (2).

By Proposition 4.13, condition (i) is equivalent to the following:

(i) The morphism $\nu \mid V^{\natural}: V^{\natural} \rightarrow\left(I_{o} N_{\mathbf{a}}\right)_{0}^{c i}$ is dominant.

On the other hand, since $\delta_{(g, h)}$ is equal to $(d \nu)_{(g, h)}: T_{(g, h)} U \rightarrow T_{\nu(g, h)}\left(M_{\mathbf{a}}\right)_{0}$ via the natural identifications $T_{(g, h)} U \cong U$ and $T_{\nu(g, h)}\left(M_{\mathbf{a}}\right)_{0} \cong\left(M_{\mathbf{a}}\right)_{0}$, condition (ii) is equivalent to the following:

(ii)' The morphism $\nu \mid V: V \rightarrow\left(I_{o} N_{\mathbf{a}}\right)_{0}$ is dominant.

Since $\left(I_{o} N_{\mathbf{a}}\right)_{0}^{c i}$ is Zariski open dense in $\left(I_{o} N_{\mathbf{a}}\right)_{0}$, the implication (i) $\Rightarrow$ (ii) is obvious. Since $V^{\natural}$ is Zariski open in $V$, the implication (ii) $\Rightarrow$ (i) follows if we show that $V^{\natural}$ is non-empty under condition (ii). Suppose that condition (ii) is fulfilled. Let $(g, h)$ be a general element of $V$. Since $g$ is general in $\left(I_{o} M_{\mathbf{b}}\right)_{0}$, the ideal $J_{g}$ defines a complete intersection of multi-degree $\mathbf{b}$ passing through $o$, and $\mu(g)$ is of rank $s$. By (ii $)^{\prime}, h(g)$ is a general element of $\left(I_{o} N_{\mathbf{a}}\right)_{0}$, and hence $J_{h(g)}$ defines a complete intersection of multi-degree a passing through $o$ and singular at $o$. Thus we have $(g, h) \in V^{\natural}$.

5. Proof of Main Theorem. First we prepare two easy lemmas.

Let $L_{1}$ and $L_{2}$ be finite-dimensional vector spaces, and let $\operatorname{Hom}\left(L_{1}, L_{2}\right)$ be the vector space of linear maps from $L_{1}$ to $L_{2}$. For $\varphi \in \operatorname{Hom}\left(L_{1}, L_{2}\right)$, we have a canonical identification

$$
T_{\varphi} \operatorname{Hom}\left(L_{1}, L_{2}\right) \cong \operatorname{Hom}\left(L_{1}, L_{2}\right) .
$$


Let $S_{k}$ be the closed subscheme of $\operatorname{Hom}\left(L_{1}, L_{2}\right)$ defined as common zeros of all $(k+1)$ minors of the matrices expressing the linear maps in terms of certain bases of $L_{1}$ and $L_{2}$.

Lemma 5.1. Let $\varphi_{0}$ be a point of $S_{k} \backslash S_{k-1}$. An element $\varphi$ of $\operatorname{Hom}\left(L_{1}, L_{2}\right)$ is contained in the subspace $T_{\varphi_{0}} S_{k}$ of $T_{\varphi_{0}} \operatorname{Hom}\left(L_{1}, L_{2}\right)$ under the identification (5.1) if and only if $\varphi\left(\operatorname{Ker} \varphi_{0}\right)$ is contained in $\operatorname{Im} \varphi_{0}$.

Proof. We can choose bases of $L_{1}$ and $L_{2}$ in such a way that $\varphi_{0}$ is expressed by the matrix $\left(\begin{array}{c|c}I_{k} & O \\ \hline O & O\end{array}\right)$. Suppose that $\varphi$ is expressed by the matrix $\left(\begin{array}{l|l}A & B \\ \hline C & D\end{array}\right)$ under these bases. Then $\varphi$ is contained in $T_{\varphi_{0}} S_{k}$ under the identification (5.1) if and only if the matrix $\left(\begin{array}{c|c}I_{k}+\varepsilon A & \varepsilon B \\ \hline \varepsilon C & \varepsilon D\end{array}\right)$ is of rank $k$, where $\varepsilon$ is the dual number; $\varepsilon^{2}=0$. This matrix is of rank $k$ if and only if $D=0$, which is equivalent to $\varphi\left(\operatorname{Ker} \varphi_{0}\right) \subseteq \operatorname{Im} \varphi_{0}$.

Let $X$ and $Y$ be connected complex manifolds, $Z$ an irreducible locally closed analytic subspace of $Y, \psi: X \rightarrow Y$ a holomorphic map, and $p$ a point of $\psi^{-1}(Z)$.

LEMMA 5.2. Suppose that $Z$ is smooth at $\psi(p)$, and that

$$
T_{\psi(p)} Z \cap \operatorname{Im}(d \psi)_{p}=0 \quad \text { and } \quad T_{\psi(p)} Z+\operatorname{Im}(d \psi)_{p}=T_{\psi(p)} Y .
$$

Then $\psi^{-1}(Z)$ is smooth at $p$. Moreover, the dimension of $\psi^{-1}(Z)$ at $p$ is equal to $\operatorname{dim} X-\operatorname{dim} Y+\operatorname{dim} Z$.

Proof. By (5.2), we have $T_{p} \psi^{-1}(Z)=(d \psi)_{p}^{-1}\left(T_{\psi(p)} Z\right)=\operatorname{Ker}(d \psi)_{p}$, and hence

$$
\operatorname{dim} T_{p} \psi^{-1}(Z)=\operatorname{dim} T_{p} X-\operatorname{dim} \operatorname{Im}(d \psi)_{p}=\operatorname{dim} X-\operatorname{dim} Y+\operatorname{dim} Z .
$$

On the other hand, the codimension of $\psi^{-1}(Z)$ in $X$ at $p$ is less than or equal to the codimension of $Z$ in $Y$ at $\psi(p)$. Combining these facts, we get the hoped-for results.

From now on, we assume that $(n, \mathbf{a}, \mathbf{b})$ satisfies the conditions required in Main Theorem. In particular, the morphism $\alpha \mid \Xi_{o}: \Xi_{o} \rightarrow \Gamma_{o}$ is dominant by Remark 4.12. Let $\Xi_{o}^{\prime}$ be the unique irreducible component of $\Xi_{o}$ that is mapped dominantly onto $\Gamma_{o}$ by $\alpha \mid \Xi_{o}$ (see Proposition 4.14).

Proposition 5.3. Let $Q=(o,\langle g\rangle,\langle f\rangle)$ be a general point of $\Xi_{o}^{\prime}$. Then:

(1) The morphism $\rho: F_{\mathbf{b}, \mathbf{a}} \rightarrow H_{n, \mathbf{a}}$ is dominant.

(2) The kernel of $(d \alpha)_{Q}: T_{Q} \widetilde{\mathcal{Z}} \rightarrow T_{\alpha(Q)} \mathcal{X}$ is of dimension equal to

$$
\operatorname{dim} F_{\mathbf{b}, \mathbf{a}}-\operatorname{dim} H_{n, \mathbf{a}}-m+2 l .
$$

(3) The image of $(d \rho)_{\pi(Q)}: T_{\pi(Q)} F_{\mathbf{b}, \mathbf{a}} \rightarrow T_{\langle f\rangle} H_{n, \mathbf{a}}$ is of codimension 1 .

Proof. First of all, note that $F_{\mathbf{b}, \mathbf{a}}$ is non-empty because $\Xi_{o}$ is non-empty. Note also that $V^{\natural}$ is non-empty by Proposition 4.13, and hence is Zariski open dense in $V$. By Proposition 4.14, the general point $Q$ of $\Xi_{o}^{\prime}$ is the image of a general point of $V^{\natural}$ by $\xi: V^{\natural} \rightarrow \Xi_{o}$. Therefore we can choose a general point $(g, h)$ of $V$ first, and then put $Q:=\xi(g, h)=(o,\langle g\rangle,\langle h(g)\rangle)$.

We start with the proof of (3). By Proposition 4.15, we have

$$
\delta_{(g, h)}(V)=\left(J_{g} N_{\mathbf{a}}+I_{o} h\left(M_{\mathbf{b}}\right)\right)_{0}=\left(I_{o} N_{\mathbf{a}}\right)_{0} .
$$


In particular, we have

$$
\operatorname{dim}\left(M_{\mathbf{a}} /\left(J_{g} N_{\mathbf{a}}+I_{o} h\left(M_{\mathbf{b}}\right)\right)\right)_{0}=n+r
$$

By Corollary $4.7(2)$, to arrive at $\operatorname{dim} \operatorname{Coker}(d \rho)_{\pi(Q)}=1$, all we have to show is

$$
\operatorname{dim}\left(M_{\mathbf{a}} /\left(J_{g} M_{\mathbf{a}}+h\left(M_{\mathbf{b}}\right)\right)\right)_{0}=1
$$

which is equivalent to

$$
\operatorname{dim} \delta_{(g, h)}(U) / \delta_{(g, h)}(V)=n+r-1,
$$

because of $(4.16),(4.17)$ and (5.4). We define a linear map $\tilde{\lambda}_{r}:\left(M_{\mathbf{a}}\right)_{0} \rightarrow \mathbb{C}^{r} \times \mathbb{C}^{n}$ by

$$
\tilde{\lambda}_{r}(f):=\left(\left(f_{1}(o), \ldots, f_{r}(o)\right),\left(\frac{\partial f_{r}}{\partial x_{1}}(o), \ldots, \frac{\partial f_{r}}{\partial x_{n}}(o)\right)\right) .
$$

Then $\delta_{(g, h)}(V)=\left(I_{\tilde{\tau}} N_{\mathbf{a}}\right)_{0}=\operatorname{Ker} \tilde{\lambda}_{r}$ holds from (5.3). Moreover, we have $\delta_{(g, h)}(U) \subseteq\left(N_{\mathbf{a}}\right)_{0}$ by $(4.18)$, and $\operatorname{dim} \tilde{\lambda}_{r}\left(\left(N_{\mathbf{a}}\right)_{0}\right)=n+r-1$. Therefore the equality (5.5) and each of the following two conditions are equivalent to one another:

$$
\begin{gathered}
\delta_{(g, h)}(U)=\left(N_{\mathbf{a}}\right)_{0}, \\
\operatorname{dim} \tilde{\lambda}_{r}\left(\delta_{(g, h)}(U)\right) \geq n+r-1 .
\end{gathered}
$$

We will prove (5.5) by showing that the inequality (5.7) holds. For $\nu=1, \ldots, s$, we define $\gamma^{(\nu)}=\left(\gamma_{1}^{(\nu)}, \ldots, \gamma_{s}^{(\nu)}\right)^{T} \in\left(M_{\mathbf{b}}\right)_{0}$ and $\eta^{(\nu)}=\left(\eta_{i j}^{(\nu)}\right) \in \operatorname{Hom}\left(M_{\mathbf{b}}, M_{\mathbf{a}}\right)_{0}$ by

$$
\gamma_{j}^{(\nu)}:=\left\{\begin{array}{ll}
0 & \text { if } j \neq \nu \\
x_{0}^{b_{j}} & \text { if } j=\nu
\end{array} \text { and } \quad \eta_{i j}^{(\nu)}:= \begin{cases}0 & \text { if }(i, j) \neq(r, \nu) \\
x_{0}^{a_{r}-b_{\nu}} & \text { if }(i, j)=(r, \nu) .\end{cases}\right.
$$

Note that $a_{r} \geq b_{\nu}$ by the condition (2.2). We then define $v^{(\nu)}, w^{(\nu)} \in \delta_{(g, h)}(U)$ by

$$
\begin{aligned}
v^{(\nu)} & :=\delta_{(g, h)}\left(\gamma^{(\nu)}, 0\right)=h\left(\gamma^{(\nu)}\right), \\
w^{(\nu)}: & :=\delta_{(g, h)}\left(0, \eta^{(\nu)}\right)=\eta^{(\nu)}(g)=\left(0, \ldots, 0, g_{\nu} x_{0}^{a_{r}-b_{\nu}}\right)^{T} .
\end{aligned}
$$

Then we have

$$
\begin{aligned}
& \tilde{\lambda}_{r}\left(v^{(\nu)}\right)=\left(\left(h_{1 \nu}(o), \ldots, h_{r \nu}(o)\right),\left(\frac{\partial h_{r \nu}}{\partial x_{1}}(o), \ldots, \frac{\partial h_{r \nu}}{\partial x_{n}}(o)\right)\right), \\
& \tilde{\lambda}_{r}\left(w^{(\nu)}\right)=\left((0, \ldots, 0),\left(\frac{\partial g_{\nu}}{\partial x_{1}}(o), \ldots, \frac{\partial g_{\nu}}{\partial x_{n}}(o)\right)\right) .
\end{aligned}
$$

In order to prove (5.7), it is enough to show that the vectors $\tilde{\lambda}_{r}\left(v^{(\nu)}\right)$ and $\tilde{\lambda}_{r}\left(w^{(\nu)}\right)$ span a hyperplane in $\mathbb{C}^{r} \times \mathbb{C}^{n}$. Since $(g, h)$ is general in $V$, the coefficients $h_{i \nu}(o), \partial h_{r \nu} / \partial x_{j}(o)$ and $\partial g_{\nu} / \partial x_{j}(o)$ of the homogeneous polynomials $g_{\nu}$ and $h_{i \nu}$ that appear in (5.8) are general except for the following restrictions:

$$
\begin{gathered}
h_{r \nu}(o)=0 \quad(1 \leq \nu \leq s), \quad h_{i \nu}(o)=0 \quad \text { if } a_{i}<b_{\nu}, \\
\frac{\partial h_{r \nu}}{\partial x_{j}}(o)=0 \quad(1 \leq j \leq n) \quad \text { if } a_{r}=b_{\nu} .
\end{gathered}
$$

Let $\Lambda$ be the $2 s \times(n+r)$ matrix whose row vectors are $\tilde{\lambda}_{r}\left(v^{(\nu)}\right)$ and $\tilde{\lambda}_{r}\left(w^{(\nu)}\right)$. Then $\Lambda$ is of the shape depicted in Figure 2, in which the entries in the submatrices marked with $*$ are general, and the $(\nu, i)$-component in the submatrix marked with $\sharp$ is general except for the restriction that it must be zero if $a_{i}<b_{\nu}$. Since the rank of a matrix is a lower 
semi-continuous function of entries, in order to prove that $\Lambda$ is of rank $n+r-1$, it is enough to show that there exists at least one matrix of rank $n+r-1$ with the shape Figure 2. Condition (2.2) implies that $r-t_{a} \leq s-t_{b}$ holds, and that the $(i, i)$-component of the submatrix $\sharp$ is subject to no restrictions for $i=1, \ldots, r-t_{a}$. Condition (2.3) implies $n+r<2 s$ and $n+r+t_{b}-t_{a} \leq 2 s$. Therefore we can define a $2 s \times(n+r)$ matrix $C$ of the shape Figure 2 by Table 1, where $c_{i}$ is the $i$-th column vector of $C$ and $e_{\mu}$ is the column vector of dimension $2 s$ whose $\nu$-th component is $\delta_{\mu \nu}$ (Kronecker's delta symbol). It is easy to see that $C$ is of rank $n+r-1$. Hence (5.7), and also (5.5) and (5.6), are proved.

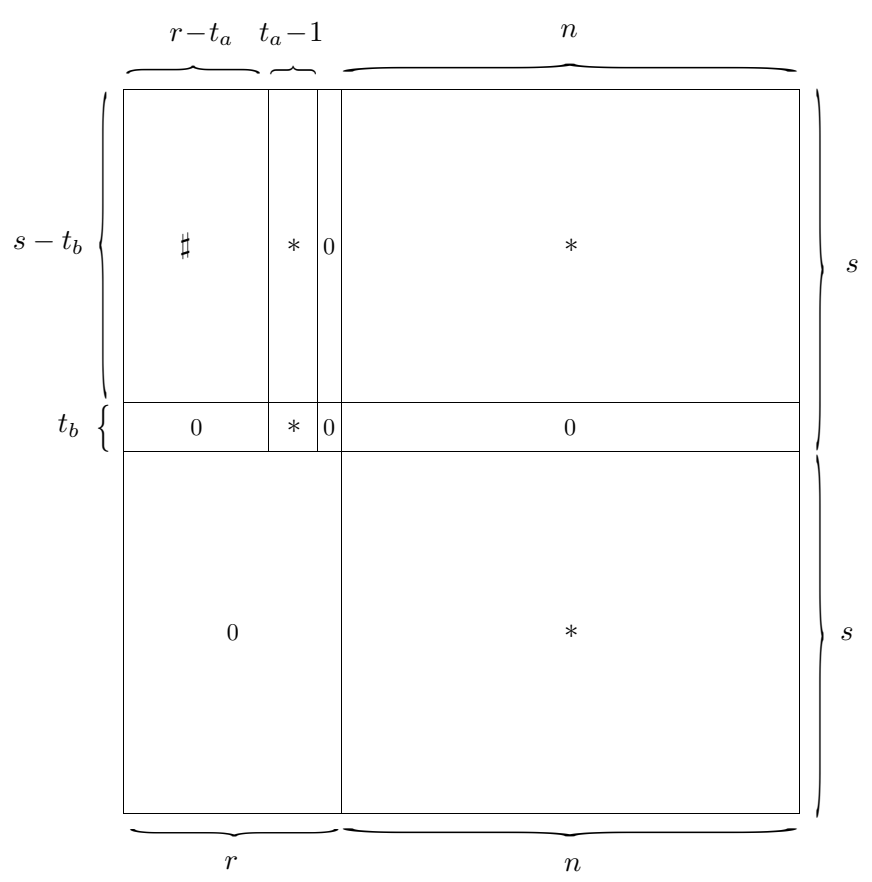

Figure 2. The shape of a $2 s \times(n+r)$ matrix

Next we prove (1). Since both of $F_{\mathbf{b}, \mathbf{a}}$ and $H_{n, \mathbf{a}}$ are smooth and irreducible, it is enough to show that the morphism $\rho: F_{\mathbf{b}, \mathbf{a}} \rightarrow H_{n, \mathbf{a}}$ is a submersion at a general point of $F_{\mathbf{b}, \mathbf{a}}$. By Corollary $4.7(2)$, it is therefore enough to prove that the following equality holds for a general $(\tilde{g}, \tilde{h}) \in U$ :

$$
\operatorname{dim}\left(M_{\mathbf{a}} /\left(J_{\tilde{g}} M_{\mathbf{a}}+\tilde{h}\left(M_{\mathbf{b}}\right)\right)\right)_{0}=0
$$

Since the left-hand side of (5.9) is an upper semi-continuous function of $(\tilde{g}, \tilde{h}) \in U$, it suffices to show that there exists at least one $(\tilde{g}, \tilde{h}) \in U$ for which $(5.9)$ holds. We will find $(\tilde{g}, \tilde{h})$ satisfying $(5.9)$ in a small neighborhood of the chosen point $(g, h)$ in $U$. From $(5.6)$, we have

$$
\operatorname{dim}\left(M_{\mathbf{a}} /\left(J_{\tilde{g}} M_{\mathbf{a}}+\tilde{h}\left(M_{\mathbf{b}}\right)\right)\right)_{0} \leq \operatorname{dim}\left(M_{\mathbf{a}} / N_{\mathbf{a}}\right)_{0}=1
$$




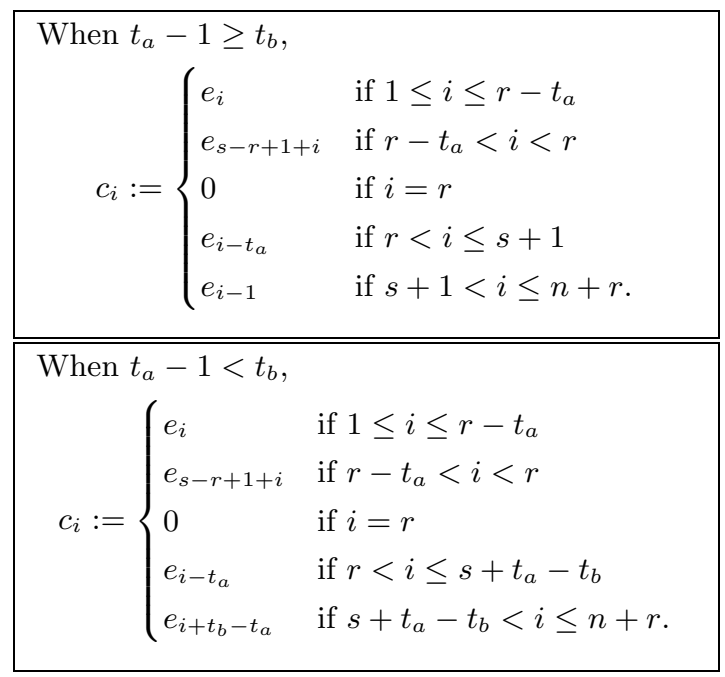

Table 1. Definition of $C$

for any $(\tilde{g}, \tilde{h})$ in a small neighborhood of $(g, h)$ in $U$. We suppose that

$$
\delta_{(\tilde{g}, \tilde{h})}(U)=\left(J_{\tilde{g}} M_{\mathbf{a}}+\tilde{h}\left(M_{\mathbf{b}}\right)\right)_{0} \text { is of codimension } 1 \text { in }\left(M_{\mathbf{a}}\right)_{0}
$$

$$
\text { for any }(\tilde{g}, \tilde{h}) \text { in a small neighborhood of }(g, h) \text { in } U \text {, }
$$

and will derive a contradiction. For a sequence $c=\left(c_{1}, \ldots, c_{s}\right)$ of complex numbers, we define $\eta^{c}=\left(\eta_{i j}^{c}\right) \in \operatorname{Hom}\left(M_{\mathbf{b}}, M_{\mathbf{a}}\right)_{0}$ by

$$
\eta_{i j}^{c}:= \begin{cases}0 & \text { if } i<r \\ c_{j} x_{0}^{a_{r}-b_{j}} & \text { if } i=r\end{cases}
$$

and consider the infinitesimal deformation $(g, h)+\varepsilon\left(0, \eta^{c}\right)$ of $(g, h)$ in $U$, where $\varepsilon$ is the dual number. Here we use the condition $a_{r} \geq b_{j}$ again. By (5.6), Lemma 5.1 and the assumption (5.10), we have

$$
\delta_{\left(0, \eta^{c}\right)}\left(\operatorname{Ker} \delta_{(g, h)}\right) \subseteq \operatorname{Im} \delta_{(g, h)}=\left(N_{\mathbf{a}}\right)_{0}
$$

for any $c$, which means that, if $(G, H) \in \operatorname{Ker} \delta_{(g, h)}$, then $\eta^{c}(G) \in\left(N_{\mathbf{a}}\right)_{0}$ for any $c$. Hence we have

$$
(G, H) \in \operatorname{Ker} \delta_{(g, h)} \Longrightarrow G \in\left(I_{o} M_{\mathbf{b}}\right)_{0} .
$$

Because (2.3) implies $2 s>n+r$, there exists a non-trivial linear relation

$$
\sum_{\nu=1}^{s} \alpha_{\nu} \tilde{\lambda}_{r}\left(v^{(\nu)}\right)+\sum_{\nu=1}^{s} \beta_{\nu} \tilde{\lambda}_{r}\left(w^{(\nu)}\right)=0 \quad\left(\alpha_{\nu}, \beta_{\nu} \in \mathbb{C}\right)
$$

among the vectors (5.8) in $\mathbb{C}^{r} \times \mathbb{C}^{n}$. Since $g$ is general in $\left(I_{o} M_{\mathbf{b}}\right)_{0}$ and $s<n$, the vectors $\tilde{\lambda}_{r}\left(w^{(\nu)}\right)(\nu=1, \ldots, s)$ are linearly independent, and hence at least one of $\alpha_{1}, \ldots, \alpha_{s}$ is 
non-zero. We put

Then we have

$$
\left(G_{1}, H_{1}\right):=\left(\sum_{\nu=1}^{s} \alpha_{\nu} \gamma^{(\nu)}, \sum_{\nu=1}^{s} \beta_{\nu} \eta^{(\nu)}\right) \in U
$$

$$
\delta_{(g, h)}\left(G_{1}, H_{1}\right)=\sum_{\nu=1}^{s} \alpha_{\nu} v^{(\nu)}+\sum_{\nu=1}^{s} \beta_{\nu} w^{(\nu)} \in \operatorname{Ker} \tilde{\lambda}_{r}=\left(I_{o} N_{\mathbf{a}}\right)_{0}=\delta_{(g, h)}(V)
$$

where the last equality follows from (5.3). Hence there exists $\left(G_{2}, H_{2}\right) \in V$ such that $\left(G_{1}-G_{2}, H_{1}-H_{2}\right) \in \operatorname{Ker} \delta_{(g, h)}$. On the other hand, since $G_{2} \in\left(I_{o} M_{\mathbf{b}}\right)_{0}$ and at least one of $\alpha_{1}, \ldots, \alpha_{s}$ is non-zero, we have $G_{1}-G_{2} \notin\left(I_{o} M_{\mathbf{b}}\right)_{0}$, which contradicts (5.11). Hence there exists a point $(\tilde{g}, \tilde{h}) \in U$ in an arbitrarily small neighborhood of $(g, h)$ such that (5.9) holds. Therefore $\rho$ is dominant.

Finally we calculate $\operatorname{dim} \operatorname{Ker}(d \alpha)_{Q}$. By Proposition 4.8, we see that $\operatorname{dim} \operatorname{Ker}(d \alpha)_{Q}$ is equal to

$$
\operatorname{dim} F_{\mathbf{b}, \mathbf{a}}-\operatorname{dim} H_{n, \mathbf{a}}-s+\operatorname{dim}\left(M_{\mathbf{a}} /\left(J_{g} M_{\mathbf{a}}+I_{o} h\left(M_{\mathbf{b}}\right)\right)\right)_{0} .
$$

The fourth term is equal to $n+r-s$ by Proposition 4.15. Since $n+r-2 s=-m+2 l$, we complete the proof of the assertion (2).

Now we are ready to prove Main Theorem.

Proof of Main Theorem. For a locally closed analytic subspace $A$ of $H_{n, \mathbf{a}}$, we denote by

$$
\begin{array}{cc}
\widetilde{\mathcal{Z}}_{A} \stackrel{\alpha_{A}}{\longrightarrow} & \mathcal{X}_{A} \\
\pi_{A} \downarrow & \\
F_{A} \underset{\rho_{A}}{\longrightarrow} & A
\end{array}
$$

the pull-back of the right square of the diagram (4.3) by $A \hookrightarrow H_{n, \mathbf{a}}$.

There exists a Zariski open dense subset $\mathcal{U}$ of $H_{n, \mathbf{a}}$ such that

$$
\begin{array}{cc}
\widetilde{\mathcal{Z}}_{\mathcal{U}} \stackrel{\alpha_{\mathcal{U}}}{\longrightarrow} \mathcal{X}_{\mathcal{U}} \\
\pi_{\mathcal{U}} \downarrow \\
F_{\mathcal{U}} \underset{\rho_{\mathcal{U}}}{\longrightarrow} & \downarrow_{\mathcal{U}}
\end{array}
$$

is locally trivial over $\mathcal{U}$ in the category of topological spaces and continuous maps, that $\phi_{\mathcal{U}}$ is smooth, and that $\rho_{\mathcal{U}}$ is smooth or $F_{\mathcal{U}}$ is empty. It is enough to show $F_{\mathbf{b}}\left(X_{b}\right) \neq \emptyset$ and $\operatorname{Im} \psi_{\mathbf{b}}\left(X_{b}\right) \supseteq V_{m}\left(X_{b}, \mathbb{Z}\right)$ for at least one point $b$ of $\mathcal{U}$, where $X_{b}$ denotes the complete intersection corresponding to a point $b$ of $\mathcal{U}$.

By Proposition 5.3 (1), $F_{\mathbf{b}}\left(X_{b}\right)$ is non-empty for any $b \in \mathcal{U}$.

By the assumption of Main Theorem, the morphism $\alpha \mid \Xi_{o}: \Xi_{o} \rightarrow \Gamma_{o}$ is dominant, and hence, by Proposition 4.14, there exists a unique irreducible component $\Xi_{o}^{\prime}$ of $\Xi_{o}$ that is mapped dominantly onto $\Gamma_{o}$ by $\alpha \mid \Xi_{o}$. Let $Q:=(o,\langle g\rangle,\langle f\rangle)$ be a general point of $\Xi_{o}^{\prime}$. Then $\alpha(Q)=(o,\langle f\rangle)$ is a general point of $\Gamma_{o}$. By Corollary 4.11, the point $o$ is the only singular point of $X_{\langle f\rangle}$, and it is a hypersurface singularity with non-degenerate Hessian. In particular, the image of $(d \phi)_{\alpha(Q)}: T_{\alpha(Q)} \mathcal{X} \rightarrow T_{\langle f\rangle} H_{n, \mathbf{a}}$ is of codimension 1 in $T_{\langle f\rangle} H_{n, \mathbf{a}}$. 
On the other hand, by Proposition $5.3(3)$, the image of $(d \rho)_{\pi(Q)}: T_{\pi(Q)} F_{\mathbf{b}, \mathbf{a}} \rightarrow T_{\langle f\rangle} H_{n, \mathbf{a}}$ is also of codimension 1 in $T_{\langle f\rangle} H_{n, \mathbf{a}}$. Hence there exists a smooth curve $C$ in $H_{n, \mathbf{a}}$ passing through $\langle f\rangle$ that satisfies

$$
\operatorname{Im}(d \phi)_{\alpha(Q)} \cap T_{\langle f\rangle} C=0, \quad \operatorname{Im}(d \rho)_{\pi(Q)} \cap T_{\langle f\rangle} C=0,
$$

and $C \cap \mathcal{U} \neq \emptyset$. We choose a sufficiently small open unit disk $\Delta$ in $C$ with the center $\langle f\rangle$, and consider the following diagrams:

$$
\begin{array}{ccccc}
\widetilde{\mathcal{Z}}_{C} \stackrel{\alpha_{C}}{\longrightarrow} \mathcal{X}_{C} & \widetilde{\mathcal{Z}}_{\Delta} \stackrel{\alpha_{\Delta}}{\longrightarrow} \mathcal{X}_{\Delta} \\
\pi_{C} \downarrow & \downarrow_{\phi} & \text { and } & \pi_{\Delta} \downarrow & \\
F_{C} \underset{\rho_{C}}{\longrightarrow} & C & & F_{\Delta} \underset{\rho_{\Delta}}{\longrightarrow} & \Delta .
\end{array}
$$

We can assume that $\Delta^{\times}:=\Delta \backslash\{\langle f\rangle\}$ is contained in $\mathcal{U}$. By Lemma 5.2 , the analytic space $\mathcal{X}_{\Delta}$ is smooth of dimension $m+1$. Moreover, the holomorphic map $\phi_{\Delta}: \mathcal{X}_{\Delta} \rightarrow \Delta$ has only one critical point, which is the point $(o,\langle f\rangle)$ on the central fiber $X_{\langle f\rangle}$, and at which the Hessian of $\phi_{\Delta}$ is non-degenerate. We select a point $b$ of $\mathcal{U}$ from $\Delta^{\times}$. Then we have a vanishing cycle $\left[\Sigma_{b}\right] \in H_{m}\left(X_{b}, \mathbb{Z}\right)$, unique up to sign, associated to the non-degenerate critical point $(o,\langle f\rangle)$ of $\phi_{\Delta}$. It is known that $V_{m}\left(X_{b}, \mathbb{Z}\right)$ is generated by $\left[\Sigma_{b}\right]$ as a module over the group ring $\mathbb{Z}\left[\pi_{1}(\mathcal{U}, b)\right]$. (See [9].) On the other hand, the image of the cylinder homomorphism $\psi_{\mathbf{b}}\left(X_{b}\right)$ is $\pi_{1}(\mathcal{U}, b)$-invariant. Therefore it is enough to show that the image of $\psi_{\mathbf{b}}\left(X_{b}\right)$ contains $\left[\Sigma_{b}\right]$.

We put

$$
O:=\pi(Q) \in F_{\mathbf{b}, \mathbf{a}}
$$

By Lemma 5.2 and (5.12), the scheme $F_{C}$ in the left diagram of (5.13) is smooth at $O$, and

$$
\operatorname{dim}_{O} F_{C}=\operatorname{dim} F_{\mathbf{b}, \mathbf{a}}-\operatorname{dim} H_{n, \mathbf{a}}+1 .
$$

From the construction of $\widetilde{\mathcal{Z}}_{C}$, we see that $\operatorname{Ker}(d \alpha)_{Q}$ is contained in the subspace $T_{Q} \widetilde{\mathcal{Z}}_{C}$ of $T_{Q} \widetilde{\mathcal{Z}}$, and that $\operatorname{Ker}(d \alpha)_{Q}$ coincides with $\operatorname{Ker}\left(d \alpha_{C}\right)_{Q}$. Hence, by Proposition $5.3(2)$, we have

$$
\operatorname{dim} \operatorname{Ker}\left(d \alpha_{C}\right)_{Q}=\operatorname{dim} F_{\mathbf{b}, \mathbf{a}}-\operatorname{dim} H_{n, \mathbf{a}}-m+2 l .
$$

Since $\widetilde{\mathcal{Z}}_{C}$ is a closed analytic subspace of $F_{C} \times \mathcal{X}_{C}$ with $\pi_{C}$ and $\alpha_{C}$ being projections, we have

$$
\operatorname{Ker}\left(d \pi_{C}\right)_{Q} \cap \operatorname{Ker}\left(d \alpha_{C}\right)_{Q}=0
$$

in $T_{Q} \widetilde{\mathcal{Z}}_{C}$. In particular, the linear map $\left(d \pi_{C}\right)_{Q}: T_{Q} \widetilde{\mathcal{Z}}_{C} \rightarrow T_{O} F_{C}$ maps $\operatorname{Ker}\left(d \alpha_{C}\right)_{Q}$ isomorphically to a linear subspace of $T_{O} F_{C}$. By the dimension counting (5.14) and (5.15), this subspace

$$
\left(d \pi_{C}\right)_{Q}\left(\operatorname{Ker}\left(d \alpha_{C}\right)_{Q}\right) \subset T_{O} F_{C}
$$

is of codimension $m-2 l+1$. Hence there exists a closed subvariety $F_{C}^{\prime}$ of $F_{C}$ with dimension $m-2 l+1$ that passes through $O$, is smooth at $O$, and satisfies

$$
T_{O} F_{C}^{\prime} \cap\left(d \pi_{C}\right)_{Q}\left(\operatorname{Ker}\left(d \alpha_{C}\right)_{Q}\right)=0 .
$$


Let us put

$$
F_{\Delta}^{\prime}:=F_{C}^{\prime} \cap F_{\Delta}, \quad \widetilde{\mathcal{Z}}_{C}^{\prime}:=\pi_{C}^{-1}\left(F_{C}^{\prime}\right) \quad \text { and } \quad \widetilde{\mathcal{Z}}_{\Delta}^{\prime}:=\pi_{\Delta}^{-1}\left(F_{\Delta}^{\prime}\right)
$$

and let

$$
\begin{aligned}
& \widetilde{\mathcal{Z}}_{C}^{\prime} \stackrel{\alpha_{C}^{\prime}}{\longrightarrow} \mathcal{X}_{C} \quad \widetilde{\mathcal{Z}}_{\Delta}^{\prime} \stackrel{\alpha_{\Delta}^{\prime}}{\longrightarrow} \mathcal{X}_{\Delta} \\
& \pi_{C}^{\prime} \downarrow \quad \downarrow \phi_{C} \text { and } \pi_{\Delta}^{\prime} \downarrow \quad \downarrow \phi_{\Delta} \\
& F_{C}^{\prime} \underset{\rho_{C}^{\prime}}{\longrightarrow} C \quad F_{\Delta}^{\prime} \underset{\rho_{\Delta}^{\prime}}{\longrightarrow} \Delta
\end{aligned}
$$

be the restriction of the diagrams (5.13). The right diagram of (5.18) is the pull-back of the left diagram of (5.18) by $\Delta \hookrightarrow C$.

Since the fiber of $\pi$ passing through $Q$ is smooth at $Q$ by the definition of $\Xi_{o}$, the holomorphic map $\pi_{\Delta}^{\prime}$ is also smooth at $Q$. Moreover, from (5.16) and (5.17), we have

$$
\operatorname{Ker}\left(d \alpha_{\Delta}^{\prime}\right)_{Q}=T_{Q} \widetilde{\mathcal{Z}}_{\Delta}^{\prime} \cap \operatorname{Ker}\left(d \alpha_{\Delta}\right)_{Q}=\left(d \pi_{C}\right)_{Q}^{-1}\left(T_{O} F_{C}^{\prime}\right) \cap \operatorname{Ker}\left(d \alpha_{C}\right)_{Q}=0 .
$$

Therefore $\alpha_{\Delta}^{\prime}$ is an immersion at $Q$. We have $\operatorname{dim} F_{\Delta}^{\prime}=m-2 l+1$. Note that $H_{m}\left(X_{b}, \mathbb{Z}\right)$ is torsion free. Hence the right diagram of (5.18) satisfies all the conditions required in Theorem $3.1(2)$. We put

$$
F_{\Delta}^{\prime}\left(X_{b}\right):=\rho_{\Delta}^{\prime}{ }^{-1}(b), \quad Z_{\Delta}^{\prime}\left(X_{b}\right):=\pi_{\Delta}^{\prime-1}\left(F_{\Delta}^{\prime}\left(X_{b}\right)\right),
$$

and consider the family

$$
\begin{aligned}
& Z_{\Delta}^{\prime}\left(X_{b}\right) \longrightarrow X_{b} \\
& \quad \downarrow \\
& F_{\Delta}^{\prime}\left(X_{b}\right)
\end{aligned}
$$

of $l$-dimensional closed analytic subspaces of $X_{b}$. By Theorem 3.1, the image of the cylinder homomorphism

$$
\psi_{\mathbf{b}}^{\prime}\left(X_{b}\right): H_{m-2 l}\left(F_{\Delta}^{\prime}\left(X_{b}\right), \mathbb{Z}\right) \rightarrow H_{m}\left(X_{b}, \mathbb{Z}\right)
$$

associated with the family (5.19) contains the vanishing cycle $\left[\Sigma_{b}\right] \in H_{m}\left(X_{b}, \mathbb{Z}\right)$. By the construction, $\psi_{\mathbf{b}}^{\prime}\left(X_{b}\right)$ is the composite of the homomorphism

$$
H_{m-2 l}\left(F_{\Delta}^{\prime}\left(X_{b}\right), \mathbb{Z}\right) \rightarrow H_{m-2 l}\left(F_{\mathbf{b}}\left(X_{b}\right), \mathbb{Z}\right)
$$

induced from the inclusion $F_{\Delta}^{\prime}\left(X_{b}\right) \hookrightarrow F_{\mathbf{b}}\left(X_{b}\right)$ and the original cylinder homomorphism $\psi_{\mathbf{b}}\left(X_{b}\right)$. Hence the image of $\psi_{\mathbf{b}}\left(X_{b}\right)$ contains $\left[\Sigma_{b}\right]$.

We put

$$
F_{C}^{\prime}\left(X_{b}\right):=\rho_{C}^{\prime-1}(b),
$$

and let $F_{C}^{\prime \prime}\left(X_{b}\right)$ be the union of irreducible components of $F_{C}^{\prime}\left(X_{b}\right)$ with dimension $m-2 l$. Then $F_{C}^{\prime \prime}\left(X_{b}\right)$ contains an $(m-2 l)$-dimensional sphere representing the vanishing cycle $\left[\sigma_{b}\right] \in H_{m-2 l}\left(F_{\Delta}^{\prime}\left(X_{b}\right), \mathbb{Z}\right)$ associated to the non-degenerate critical point $O$ of $\rho_{\Delta}^{\prime}$. Let $T$ be the Zariski closure of $\alpha_{C}^{\prime}\left(\pi_{C}^{\prime-1}\left(F_{C}^{\prime \prime}\left(X_{b}\right)\right)\right)$ in $X_{b}$. Then $T$ is of dimension $m-l$, and $\left[\Sigma_{b}\right]= \pm \psi_{\mathbf{b}}^{\prime}\left(X_{b}\right)\left(\left[\sigma_{b}\right]\right)$ is represented by a topological cycle whose support is contained in $T$. Therefore we obtain the following: 
Corollary 5.4. Suppose that ( $n, \mathbf{a}, \mathbf{b})$ satisfies the conditions of Main Theorem. Let $X$ be a general complete intersection of multi-degree $\mathbf{a}$ in $\mathbb{P}^{n}$. Then every vanishing cycle of $X$ is represented by a topological cycle whose support is contained in a Zariski closed subset of $X$ with codimension $l$.

6. Gröbner bases method. Suppose we are given a triple $(n, \mathbf{a}, \mathbf{b})$ that satisfies the conditions (2.2) and (2.3) of Main Theorem. We will describe a method to determine whether this triple satisfies the second condition of Main Theorem.

First we choose a prime integer $p$, and put

$$
R^{(p)}:=\mathcal{F}_{p}\left[x_{0}, \ldots, x_{n}\right] .
$$

We define graded $R^{(p)}$-modules $M_{\mathbf{a}}^{(p)}, M_{\mathbf{b}}^{(p)}, N_{\mathbf{a}}^{(p)}$, and ideals $I_{o}^{(p)}, J_{g}^{(p)}$ of $R^{(p)}$ in the same way as in Section 4 except for the coefficient field. We generate an element $g=$ $\left(g_{1}, \ldots, g_{s}\right)^{T}$ of $\left(I_{o}^{(p)} M_{\mathbf{b}}^{(p)}\right)_{0}$ and a homomorphism $h=\left(h_{i j}\right) \in \operatorname{Hom}\left(M_{\mathbf{b}}^{(p)}, N_{\mathbf{a}}^{(p)}\right)_{0}$ in a random way. Then we can calculate

$$
\operatorname{dim}_{\mathcal{F}_{p}}\left(M_{\mathbf{a}}^{(p)} /\left(J_{g}^{(p)} M_{\mathbf{a}}^{(p)}+I_{o}^{(p)} h\left(M_{\mathbf{b}}^{(p)}\right)\right)\right)_{0}
$$

by means of Gröbner bases. If this dimension is not greater than $n+r-s$, then condition (iv) of Proposition 4.15 is fulfilled, because this condition is an open condition. Hence the morphism $\alpha \mid \Xi_{o}: \Xi_{o} \rightarrow \Gamma_{o}$ is dominant.

7. Application of a theorem of Debarre and Manivel. From now on, we use the following terminology. A sequence always means a finite non-decreasing sequence of positive integers. For a sequence a, let $\min (\mathbf{a})$ and $\max (\mathbf{a})$ be the first and the last elements of $\mathbf{a}$, respectively, and let $|\mathbf{a}|$ denote the length of $\mathbf{a}$. Let $\mathbf{a}^{\prime}$ be another sequence. We denote by $\mathbf{a} \uplus \mathbf{a}^{\prime}$ the sequence of length $|\mathbf{a}|+\left|\mathbf{a}^{\prime}\right|$ obtained by re-arranging the conjunction $\left(\mathbf{a}, \mathbf{a}^{\prime}\right)$ into the non-decreasing order. For an integer $a \geq 2$, we define $(a)$ ! to be the sequence $(2, \ldots, a)$ of length $a-1$, and for a sequence $\mathbf{a}=\left(a_{1}, \ldots, a_{r}\right)$ with $\min (\mathbf{a}) \geq 2$, we put

$$
\mathbf{a} !:=\left(a_{1}\right) ! \uplus \ldots \uplus\left(a_{r}\right) ! .
$$

We sometimes write a sequence by indicating the number of repetition of each integer in the sequence by a superscript. For example, we have

$$
(2,3,3,4) !=(2,2,2,2,3,3,3,4)=\left(2^{4}, 3^{3}, 4\right) .
$$

Let $n$ and $\ell$ be positive integers, and $\mathbf{a}=\left(a_{1}, \ldots, a_{r}\right)$ a sequence. According to [5], we put

$$
\delta(n, \mathbf{a}, \ell):=(\ell+1)(n-\ell)-\sum_{i=1}^{r}\left(\begin{array}{c}
a_{i}+\ell \\
\ell
\end{array}\right),
$$

and $\delta_{-}(n, \mathbf{a}, \ell):=\min \{\delta(n, \mathbf{a}, \ell), n-2 \ell-|\mathbf{a}|\}$.

Theorem 7.1 ([5], Théorème 2.1). A general complete intersection of multi-degree $\mathbf{a}$ in $\mathbb{P}^{n}$ contains an $\ell$-dimensional linear subspace if and only if $\delta_{-}(n, \mathbf{a}, \ell) \geq 0$. 
THEOREM 7.2. Let $\mathbf{a}=\left(a_{1}, \ldots, a_{r}\right)$ be a sequence satisfying

$$
\min (\mathbf{a}) \geq 2 \quad \text { and } \quad \sum a_{i} \leq n .
$$

Let $\mathbf{a}^{\prime}$ be a sub-sequence of $\mathbf{a}$ such that $\max \left(\mathbf{a}^{\prime}\right)=\max (\mathbf{a})$, and let $\mathbf{a}^{\prime \prime}$ be the complement to $\mathbf{a}^{\prime}$ in $\mathbf{a}$. (When $\mathbf{a}^{\prime}=\mathbf{a}$, the sequence $\mathbf{a}^{\prime \prime}$ is empty.) Suppose that a positive integer $\lambda$ satisfies:

$$
\delta_{-}\left(n-\left|\mathbf{a}^{\prime}\right|, \mathbf{a}^{\prime} !, \lambda-1\right) \geq 0, \quad\left|\mathbf{a}^{\prime \prime}\right|<\lambda \quad \text { and } \quad n-r>2\left(\lambda-\left|\mathbf{a}^{\prime \prime}\right|\right) .
$$

Let

$$
\mathbf{b}:=\left(1^{n-\lambda}\right) \uplus \mathbf{a}^{\prime \prime} .
$$

Then $F_{\mathbf{b}}(X)$ is non-empty for a general complete intersection $X$ of multi-degree $\mathbf{a}$ in $\mathbb{P}^{n}$, and the image of the cylinder homomorphism $\psi_{\mathbf{b}}(X)$ contains $V_{m}(X, \mathbb{Z})$.

Proof. Note that $l=n-|\mathbf{b}|$ is equal to $\lambda-\left|\mathbf{a}^{\prime \prime}\right|$. Since $\max \left(\mathbf{a}^{\prime}\right)=\max (\mathbf{a})$, we can assume that $a_{r}$ is a member of $\mathbf{a}^{\prime}$. Let $f=\left(f_{1}, \ldots, f_{r}\right)^{T}$ be a general element of $\left(I_{o} N_{\mathbf{a}}\right)_{0}$, and let $Y_{i}$ be the hypersurface of degree $a_{i}$ defined by $f_{i}=0$. We put

$$
X^{\prime}:=\bigcap_{a_{i} \in \mathbf{a}^{\prime}} Y_{i} \text { and } X^{\prime \prime}:=\bigcap_{a_{i} \in \mathbf{a}^{\prime \prime}} Y_{i} \text {. }
$$

By Proposition 4.9, $X^{\prime}$ is a general member of the family of complete intersections of multi-degree $\mathbf{a}^{\prime}$ possessing a singular point at $o$. By means of the projection with the center $o$, we see that $X^{\prime}$ contains a linear subspace of dimension $\ell>0$ that passes through $o$ if and only if a general complete intersection of multi-degree $\mathbf{a}^{\prime} !$ in $\mathbb{P}^{n-\left|\mathbf{a}^{\prime}\right|}$ contains an $(\ell-1)$-dimensional linear subspace. By Theorem 7.1, the first condition of (7.1) implies that $X^{\prime}$ contains a linear subspace $\Lambda$ of dimension $\lambda$ passing through $o$. In particular, we have $\lambda<n-\left|\mathbf{a}^{\prime}\right|$. Using this inequality and the second and the third conditions of (7.1), we can easily check that $(n, \mathbf{a}, \mathbf{b})$ satisfies the conditions (2.2) and (2.3) in Main Theorem.

We put

$$
Z:=\Lambda \cap X^{\prime \prime}
$$

Then $Z$ is a complete intersection of multi-degree $\mathbf{b}$ contained in $X_{\langle f\rangle}=X^{\prime} \cap X^{\prime \prime}$ and passing through $o$. Moreover, since the polynomials $f_{i}\left(a_{i} \in \mathbf{a}^{\prime \prime}\right)$ are general with respect to $\Lambda$, the complete intersection $Z$ is smooth. Thus the second condition of Main Theorem is also satisfied.

8. The generalized Hodge conjecture for complete intersections. Suppose we are given a pair $(n, \mathbf{a})$ satisfying $\min (\mathbf{a}) \geq 2$ and $\sum a_{i} \leq n$. We put

$$
k:=\left[\left(n-\sum a_{i}\right) / \max (\mathbf{a})\right]+1 .
$$

The Hodge structure of the middle cohomology group $H^{m}(X)$ of a general complete intersection $X$ of multi-degree a in $\mathbb{P}^{n}$ has the property (1.1). We will investigate the consequence of the generalized Hodge conjecture that there should exist a Zariski closed subset $T$ of $X$ with codimension $k$ such that every element of $H_{m}(X, \mathbb{Q})$ is represented by a topological cycle whose support is contained in $T$. Note that $H_{m}(X, \mathbb{Q})$ is generated by vanishing cycles and, if $m$ is even, the homology class of an intersection of $X$ and 


\begin{tabular}{|r|l|}
\hline$n$ & \multicolumn{1}{|c|}{$\mathbf{a}$} \\
\hline \hline 6 & $(3)$ \\
\hline 7 & $(3)$ \\
\hline 8 & $(2,3),(3),(4)$ \\
\hline 9 & $(2,3),(3),\left(3^{2}\right),(4)$ \\
\hline 10 & $(2,3),(3),\left(3^{2}\right),(4)$ \\
\hline 11 & $\left(2^{2}, 3\right),(2,4),(3),\left(3^{2}\right),(4),(5)$ \\
\hline 12 & $\left(2^{2}, 3\right),(2,3),\left(2,3^{2}\right),(2,4),(3),\left(3^{2}\right),\left(3^{3}\right),(5)$ \\
\hline 13 & $\left(2^{3}, 3\right),\left(2^{2}, 4\right),(2,3),\left(2,3^{2}\right),(2,4),(3),\left(3^{2}\right),\left(3^{3}\right),(3,4),(5)$ \\
\hline 14 & $\left(2^{3}, 3\right),\left(2^{2}, 4\right),(2,5),(3),\left(3^{2}\right),\left(3^{3}\right),(3,4),(4),\left(4^{2}\right),(5)$ \\
\hline 15 & $\left(2^{2}, 3\right),\left(2^{2}, 3^{2}\right),\left(2^{2}, 4\right),(2,3),\left(2,3^{2}\right),\left(2,3^{3}\right),(2,3,4),(2,5),(3),\left(3^{2}\right)$, \\
& $\left(3^{3}\right),\left(3^{4}\right),(4),\left(4^{2}\right),(6)$ \\
\hline 16 & $\left(2^{4}, 3\right),\left(2^{3}, 4\right),\left(2^{2}, 5\right),(2,3),\left(2,3^{2}\right),\left(2,3^{3}\right),(2,3,4),(2,5),(3),\left(3^{2}\right)$, \\
\hline 17 & $\left(3^{3}\right),\left(3^{4}\right),(3,5),(6)$ \\
\hline 18 & $\left(2^{3}, 4\right),\left(2^{2}, 5\right),(2,4),\left(2,4^{2}\right),(2,6),(3),\left(3^{2}\right),\left(3^{3}\right),\left(3^{4}\right),\left(3^{2}, 4\right),(3,5),(6)$ \\
\hline 19 & $\left(2^{4}, 4\right),\left(2^{3}, 5\right),(2,3),\left(2,3^{2}\right),\left(2,3^{3}\right),\left(2,3^{4}\right),(2,3,5),(2,6),(3),\left(3^{2}\right)$, \\
\hline 20 & $\left(3^{3}\right),\left(3^{4}\right),\left(3^{5}\right),(3,6),(7)$ \\
\hline 21 & $\left(2^{3}, 3,4\right),\left(2^{3}, 5\right),\left(2^{2}, 6\right),(3),\left(3^{2}\right),\left(3^{3}\right),\left(3^{4}\right),\left(3^{5}\right),\left(3^{2}, 5\right),(3,6),(7)$ \\
\hline 22 & $\left(2^{5}, 4\right),\left(2^{4}, 5\right),\left(2^{2}, 3,5\right),\left(2^{2}, 6\right),(2,7)$ \\
\hline 23 & $\left(2^{6}, 4\right),\left(2^{3}, 3,5\right),\left(2^{3}, 6\right),(3),\left(3^{2}\right),\left(3^{3}\right),\left(3^{4}\right),\left(3^{5}\right),\left(3^{6}\right),(3,7),(8)$ \\
\hline 24 & $\left(2^{5}, 5\right),\left(2^{2}, 3,6\right),\left(2^{2}, 7\right)$ \\
\hline 25 & $\left(2^{4}, 6\right)$ \\
\hline 26 & $\left(2^{6}, 5\right),\left(2^{3}, 7\right)$ \\
\hline 27 & $\left(2^{5}, 6\right)$ \\
\hline
\end{tabular}

Table 2. The 148 pairs

a linear subspace of $\mathbb{P}^{n}$. Hence, by Corollary 5.4, this consequence is verified if we can find $\mathbf{b}$ with the following properties:

$$
l:=n-|\mathbf{b}|=k,
$$

$(n, \mathbf{a}, \mathbf{b})$ satisfies the assumptions of Main Theorem.

In the following, we assume $m>2 k$. This inequality $m>2 k$ fails to hold if and only if $m \leq 2$ or $\mathbf{a}=(2)$ or $(\mathbf{a}=(2,2)$ and $m$ even $)$. In these cases, the Hodge conjecture has already been proved.

Proposition 8.1 ([14], [16]). If $k=1$, then $\mathbf{b}=\left(1^{n-1}\right)$ satisfies condition (8.1). If $\mathbf{a}=\left(2^{r}\right)$, then $\mathbf{b}=\left(1^{n-[n / 2]}, 2^{r-1}\right)$ satisfies condition $(8.1)$.

Proof. Put $\mathbf{a}^{\prime}=\mathbf{a}$ in the case $k=1$ and $\mathbf{a}^{\prime}=(2)$ in the case $\mathbf{a}=\left(2^{r}\right)$, and apply Theorem 7.2. 


\begin{tabular}{|l|l|l|}
\hline$n$ & \multicolumn{1}{|c|}{$\mathbf{a}$} & \multicolumn{1}{c|}{$\mathbf{b}$} \\
\hline \hline 10 & $\left(2^{2}, 3\right)$ & $\left(1^{7}, 2\right)$ \\
\hline \multirow{2}{*}{11} & $(2,3)$ & $\left(1^{7}, 2\right)$ \\
\cline { 2 - 3 } & $\left(2,3^{2}\right)$ & $\left(1^{7}, 2,3\right)$ \\
\hline 12 & $\left(2^{3}, 3\right)$ & $\left(1^{7}, 2^{3}\right)$ \\
\hline \multirow{4}{*}{13} & $\left(2^{2}, 3\right)$ & $\left(1^{8}, 2^{2}\right)$ \\
\cline { 2 - 3 } & $\left(2^{2}, 3^{2}\right)$ & $\left(1^{8}, 2^{2}, 3\right)$ \\
\hline \multirow{5}{*}{14} & $\left(2^{2}, 3\right)$ & $\left(1^{10}, 2\right)$ \\
\cline { 2 - 3 } & $\left(2^{2}, 3^{2}\right)$ & $\left(1^{9}, 2^{2}, 3\right)$ \\
\cline { 2 - 3 } & $(2,3)$ & $\left(1^{9}, 2\right)$ \\
\cline { 2 - 3 } & $\left(2,3^{2}\right)$ & $\left(1^{9}, 2,3\right)$ \\
\cline { 2 - 3 } & $\left(2,3^{3}\right)$ & $\left(1^{9}, 2,3^{2}\right)$ \\
\hline
\end{tabular}

\begin{tabular}{|l|l|l|}
\hline$n$ & \multicolumn{1}{|c|}{$\mathbf{a}$} & \multicolumn{1}{c|}{$\mathbf{b}$} \\
\hline \hline 15 & $\left(2^{4}, 3\right)$ & $\left(1^{9}, 2^{4}\right)$ \\
\hline \multirow{3}{*}{16} & $\left(2^{3}, 3\right)$ & $\left(1^{10}, 2^{3}\right)$ \\
\cline { 2 - 3 } & $\left(2^{3}, 3^{2}\right)$ & $\left(1^{10}, 2^{3}, 3\right)$ \\
\hline \multirow{4}{*}{17} & $\left(2^{3}, 3\right)$ & $\left(1^{13}, 2\right)$ \\
\cline { 2 - 3 } & $\left(2^{3}, 3^{2}\right)$ & $\left(1^{11}, 2^{3}, 3\right)$ \\
\cline { 2 - 3 } & $\left(2^{2}, 3\right)$ & $\left(1^{11}, 2^{2}\right)$ \\
\cline { 2 - 3 } & $\left(2^{2}, 3^{2}\right)$ & $\left(1^{11}, 2^{2}, 3\right)$ \\
\cline { 2 - 3 } & $\left(2^{2}, 3^{3}\right)$ & $\left(1^{11}, 2^{2}, 3^{2}\right)$ \\
\hline \multirow{4}{*}{18} & $\left(2^{2}, 3\right)$ & $\left(1^{13}, 2\right)$ \\
\cline { 2 - 3 } & $\left(2^{2}, 3^{2}\right)$ & $\left(1^{13}, 2,3\right)$ \\
\cline { 2 - 3 } & $\left(2^{2}, 3^{3}\right)$ & $\left(1^{13}, 2,3^{2}\right)$ \\
\hline
\end{tabular}

\begin{tabular}{|l|l|l|}
\hline$n$ & \multicolumn{1}{|c|}{$\mathbf{a}$} & \multicolumn{1}{c|}{$\mathbf{b}$} \\
\hline \hline \multirow{4}{*}{18} & $(2,3)$ & $\left(1^{12}, 2\right)$ \\
\cline { 2 - 3 } & $\left(2,3^{2}\right)$ & $\left(1^{12}, 2,3\right)$ \\
\cline { 2 - 3 } & $\left(2,3^{3}\right)$ & $\left(1^{12}, 2,3^{2}\right)$ \\
\cline { 2 - 3 } & $\left(2,3^{4}\right)$ & $\left(1^{12}, 2,3^{3}\right)$ \\
\hline \multirow{3}{*}{19} & $\left(2^{4}, 3\right)$ & $\left(1^{12}, 2^{4}\right)$ \\
\cline { 2 - 3 } & $\left(2^{4}, 3^{2}\right)$ & $\left(1^{12}, 2^{4}, 3\right)$ \\
\hline \multirow{2}{*}{20} & $\left(2^{3}, 3\right)$ & $\left(1^{13}, 2^{3}\right)$ \\
\cline { 2 - 3 } & $\left(2^{3}, 3^{2}\right)$ & $\left(1^{13}, 2^{3}, 3\right)$ \\
\cline { 2 - 3 } & $\left(2^{3}, 3^{3}\right)$ & $\left(1^{13}, 2^{3}, 3^{2}\right)$ \\
\hline
\end{tabular}

Table 3. Examples of triples obtained by Gröbner bases method

In these cases, the consequence of the generalized Hodge conjecture is verified in any dimension.

We have made an exhaustive search in $n \leq 40$, and found 148 pairs $(n, \mathbf{a})$ that are not covered by Proposition 8.1, but for which Theorem 7.2 yields $\mathbf{b}$ satisfying condition (8.1) by taking an appropriate sub-sequence $\mathbf{a}^{\prime}$. We list these $(n, \mathbf{a})$ in Table 2 . No such $(n, \mathbf{a})$ are found if $n>27$. Even if $(n, \mathbf{a})$ does not appear in Table 2, the calculation of the dimension (6.1) by Gröbner bases sometimes gives us b satisfying condition (8.1). Examples of these $(n, \mathbf{a}, \mathbf{b})$ in $n \leq 20$ are given in Table 3 . From these results, we can find $\mathbf{b}$ satisfying condition (8.1) for any $(n, \mathbf{a})$ with $n \leq 9$. When $n=10, \mathbf{a}=(2,4)$ and $\mathbf{a}=(5)$ appear in neither Tables 2 nor 3.

As a closing remark, let us return to the classical example of cubic threefolds $([2])$. Our method shows that, not only the family of lines $\mathbf{b}=\left(1^{3}\right)$, but also the family of curves with $\mathbf{b}=\left(1^{2}, 2\right)$ or $\left(1,2^{2}\right)$ or $\left(2^{3}\right)$ give a surjective cylinder homomorphism on the middle homology group $H_{3}(X, \mathbb{Z})$ of a general cubic threefold $X$.

Acknowledgments. Part of this work was done during the author's stay at Nagoya University in December 2000. He would like to thank Professors Shigeyuki Kondo and Hiroshi Saito for their warm hospitality.

\section{References}

[1] A. Beauville, R. Donagi, La variété des droites d'une hypersurface cubique de dimension 4, C. R. Acad. Sci. Paris Sér. I Math. 301 (1985), 703-706.

[2] C. H. Clemens, P. A. Griffiths, The intermediate Jacobian of the cubic threefold, Ann. of Math. (2) 95 (1972), 281-356.

[3] H. Clemens, On the surjectivity of Abel-Jacobi mappings, Ann. of Math. (2) 117 (1983), 71-76. 
[4] A. Collino, The Abel-Jacobi isomorphism for the cubic fivefold, Pacific J. Math. 122 (1986), $43-55$.

[5] O. Debarre, L. Manivel, Sur la variété des espaces linéaires contenus dans une intersection complète, Math. Ann. 312 (1998), 549-574.

[6] P. Deligne, Cohomologie des intersections complètes, in: Groupes de monodromie en géométrie algébrique. Séminaire de Géométrie Algébrique du Bois-Marie 1967-1969 (SGA 7 II) par P. Deligne et N. Katz, Exposé XI, Lecture Notes Math. 340, Springer, Berlin, 1973, 39-61.

[7] M. Golubitsky, V. Guillemin, Stable Mappings and their Singularities, Graduate Texts in Math. 14, Springer, New York, 1973.

[8] A. Grothendieck, Hodge's general conjecture is false for trivial reasons, Topology 8 (1969), 299-303.

[9] K. Lamotke, The topology of complex projective varieties after S. Lefschetz, Topology 20 (1981), 15-51.

[10] M. Letizia, The Abel-Jacobi mapping for the quartic threefold, Invent. Math. 75 (1984), 477-492.

[11] J. D. Lewis, The cylinder homomorphism associated to quintic fourfolds, Compositio Math. 56 (1985), 315-329.

[12] J. D. Lewis, The cylinder correspondence for hypersurfaces of degree $n$ in $\mathbb{P}^{n}$, Amer. J. Math. 110 (1988), 77-114.

[13] L. Picco Botta, On the intersection of three quadrics, J. Reine Angew. Math. 399 (1989), 188-207.

[14] I. Shimada, On the cylinder homomorphisms of Fano complete intersections, J. Math. Soc. Japan 42 (1990), 719-738.

[15] I. Shimada, On the cylinder isomorphism associated to the family of lines on a hypersurface, J. Fac. Sci. Univ. Tokyo Sect. IA Math. 37 (1990), 703-719.

[16] I. Shimada, On the cylinder homomorphism for a family of algebraic cycles, Duke Math. J. 64 (1991), 201-205.

[17] I. Shimada, Grothendieck's generalized Hodge conjecture for certain Fano varieties, in: Algebraic Cycles and Related Topics (Kitasakado, 1994), World Sci. Publishing, River Edge, 1995, 51-67.

[18] J. H. M. Steenbrink, Some remarks about the Hodge conjecture, in: Hodge Theory (Sant Cugat, 1985), Lecture Notes in Math. 1246, Springer, Berlin, 1987, 165-175.

[19] T. Terasoma, Hodge conjecture for cubic 8-folds, Math. Ann. 288 (1990), 9-19.

[20] R. Thom, Quelques propriétés globales des variétés différentiables, Comment. Math. Helv. 28 (1954), 17-86.

[21] G. E. Welters, Abel-Jacobi Isogenies for Certain Types of Fano Threefolds, Math. Centre Tracts 141, Mathematisch Centrum, Amsterdam, 1981. 